\title{
Synthesis and Methodology for Optimal Design of a Parallel Remote Center of Motion Mechanism: Application to Robotic Eye Surgery
}

Jonas Smits, Dominiek Reynaerts, Emmanuel Vander Poorten

(Accepted pre-print)

\section{Accepted for:}

Mechanism and Machine Theory (Elsevier)

Submitted: 03/12/2019

Accepted: $25 / 03 / 2020$

\section{Citation}

Smits, J., Reynaerts, D., \& Vander Poorten, E. (2020). Synthesis and methodology for optimal design of a parallel remote center of motion mechanism: Application to robotic eye surgery, Mechanism and Machine Theory, https://doi.org/10.1016/j.mechmachtheory.2020.103896

\section{Copyright statement}

Elsevier Green Open Access, Accepted Manuscript

(C)2020. This manuscript version is made available under the CC-BY-NC-ND 4.0 license http://creativecommons.org/licenses/by-nc-nd/4.0/ 


\title{
Synthesis and Methodology for Optimal Design of a Parallel Remote Center of Motion Mechanism: Application to Robotic Eye Surgery
}

\author{
Jonas Smits*, Dominiek Reynaerts, Emmanuel Vander Poorten \\ Department of Mechanical Engineering - Robot-Assisted Surgery Group \\ KU Leuven - University of Leuven, 3001 Heverlee, Belgium
}

Accepted preprint

Mechanism and Machine Theory (Elsevier)

submitted 03/12/2019, accepted 25/03/2020

https://doi.org/10.1016/j.mechmachtheory.2020.103896

\begin{abstract}
This work reports on the synthesis of a parallel Remote Center of Motion (RCM) mechanism, and an optimized design for the use-case of robot-assisted vitreoretinal surgery. A 2-DoF planar RCM mechanism is proposed and synthesised as part of a $4-\mathrm{DoF}$ RCM mechanism. The proposed design substantially reduces the occupied volume at the end-effector. This solves a major problem present in related state-of-the-art, which poses limitations on sterile end-effector design and surgical instrument compatibility. Subsequently, an optimal design algorithm is proposed and implemented for the given use-case. The workspace is determined within mechanism constraints, after which performance parameters such as workspace coverage, potential energy, manipulability, reflected stiffness are determined for specific areas of interest within a desired workspace. Subsequently, these parameters are combined in a score function identifying an optimal kinematic design. When compared with the closely-related prior art, the resulting design shows improvements in relevant workspace coverage, reduced gravity compensation effort, and more isotropic manipulability. Overall reflected stiffness is reduced and should be taken into account in future design phases. Future work includes the integration of the kinematic design into a detailed conceptual design and a first prototype development.
\end{abstract}

Keywords: Mechanism design, Kinematic analysis, Remote center of motion, Design optimisation, Optimal design algorithm, Minimally invasive surgery, Surgical robotics, Co-manipulation

\section{Introduction}

During Minimally Invasive Surgery (MIS), relatively long and thin surgical instruments are inserted through small incisions in the body. These instruments are manipulated through and about the incision throughout the procedure. When compared to open surgery, patients undergoing MIS benefit from shorter 5 recovery times and reduced post-operational complications [1, 2. However, this is paired with additional challenges. Surgeons are faced with limited dexterity and vision, as they are required to manipulate the instruments using handles present outside the body while observing the instrument tips inside the body via an inserted surgical camera. Furthermore, this challenging environment makes it easy to overlook the forces occurring at the incisions in the body wall, which act as pivot points for the instruments. As such, large interaction forces at the incision site may lead to adverse effects for the patient. In order to aid the surgeon and limit tissue interaction forces a Remote Center of Motion (RCM), being a restriction of

\footnotetext{
${ }^{*}$ Corresponding author

Email address: jonas.smits@kuleuven.be (Jonas Smits)
} 
instrument motion about a fixed point in space without the presence of a physical joint, has been implemented advantageously. Most notable in surgical robotic devices, instrument motion is typically restricted to four Degrees of Freedom (DoF) through and about the incision. More specifically, the RCM combines one

15 translational DoF for instrument insertion or retraction with three rotational DoF about the RCM. In doing so, forces on the tissue surrounding the incision can be limited.

\subsection{Remote center of motion (RCM) mechanisms}

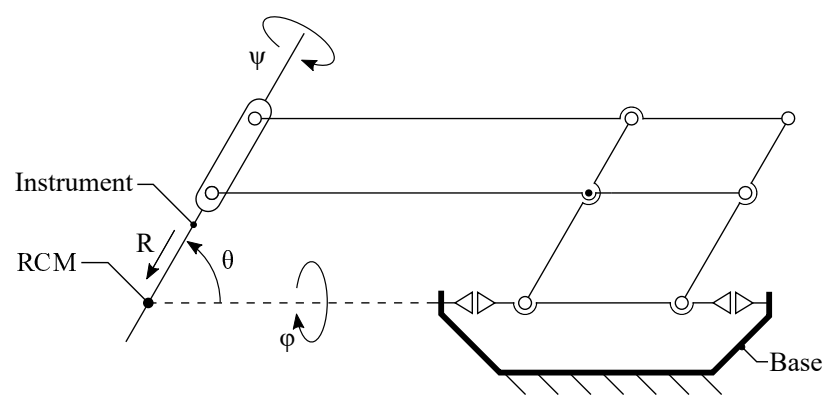

Figure 1: An annotated example of a parallelogram-based RCM mechanism. The shown mechanism is a serial combination of a linear stage providing $R$, a double parallelogram mechanism providing $\theta$, and a rotary joint providing $\phi$. A 4 th rotational DoF $\psi$ is defined as a rotation about the instrument's longitudinal axis.

Generally, the RCM is either provided relying on software-based kinematic constraints or provided inherently via a dedicated RCM mechanism. Systems using an RCM mechanism provide increased safety and reliability, as they do not rely on synchronous multi-DoF robot control for maintaining this kinematic constraint. A broad variety of RCM mechanisms for MIS have been described in the literature. The interested reader is referred to 3 for a detailed overview of RCM mechanisms in MIS.

A notable subgroup is parallelogram-based RCM mechanisms (Fig. 1). First introduced by Taylor et al. 4, several applications of parallelogram-based mechanisms in surgical robotics have been reported since 5, 6, 7. Often, the requirement of adding a translational DoF at the end-effector is present. This is done by adding a linear actuator at the proximal end of the mechanism, in near vicinity with the patient. However, this can be seen as problematic for MIS applications for the following reasons. In general, placement of actuation is preferably as far away from the surgical scene as possible, both for improving patient safety as well as to avoid interference with peripheral equipment such as imaging devices. In addition, a voluminous end-effector may limit robot movement in already confined areas, as is the case when using a surgical microscope during microsurgery. Furthermore, the added mass at the proximal end of the mechanism may pose limitations on controller bandwidth and stability. As an alternative to a proximal linear actuator, this translational DoF also may be implemented via a proximal translational joint in combination with pulleys and cables, allowing the actuator to be placed closer to the base 8 . While feasible, this introduces a number of additional design concerns such as limited transmission stiffness, design complexity, added friction, and increased maintenance.

\subsection{RCM Mechanisms for robot-assisted vitreoretinal surgery}

While these concerns are related to various types of robot-assisted MIS, the concern of end-effector volume is arguably most relevant in highly confined areas with surgery being performed on a small scale. Such is

40 the case during robot-assisted vitreoretinal surgery, which forms the use-case for this work. This type of MIS is performed minimally invasive via an incision in the eyewall. As such, an RCM can be advantageously implemented to prevent excessive forces at the incision site. Not doing so risks unintentionally damaging the eye anatomy, and may cause unwanted eye movements during surgery which poses additional challenges for high-precision interactions with the retina. The interested reader is referred to [9] for a general overview 45 of robot-assisted retinal surgery. 
Within this field, a variety of RCM mechanisms have been proposed 10. Several designs are based on a circular arc guide rail for planar rotation, followed by a rotary joint with its axis coinciding with the planar point of rotation [11, 12, 13. Other designs utilize parallel linkage combinations - such as the prior mentioned dual parallelogram - for planar rotation, followed by a rotary joint with its axis coinciding with the planar point of rotation 14, 15. A more recent conceptual design relies on curved linkages to provide two coupled rotations about an RCM [16]. These RCM mechanisms generally only provide rotations and therefore require the presence of an additional proximal actuator in close vicinity of the patient for providing the translational instrument DoF. Alternatively, some designs incorporate an RCM mechanism able to provide some of the RCM DoFs, while relying on active motion control for the remaining RCM DoFs [17. Similar to

${ }_{55}[18,19,20,21,22$, these solutions provide a virtual RCM - opposed to a mechanical RCM - and are therefore not further considered in this work. Within this field, the only exception known to be able to provide an $\mathrm{RCM}$ without the need for a proximal linear actuator or reliance on control algorithms are 2-DoF planar RCM mechanisms. Such mechanisms are currently used in the robotic technology of two groups working on robot-assisted vitreoretinal surgery [23, 24]. What follows is a more detailed review of this class of RCM 60 mechanisms.

\subsection{2-DoF planar RCM mechanisms}

In order to omit the need for proximal actuation, the class of 2-DoF planar RCM mechanisms has been proposed which couple both planar RCM DoF $R$ and $\theta$, allowing for the translational DoF $R$ to be actuated distally via coupled DoF of the mechanism. As such, the concerns of a voluminous and heavy end-effector 65 can be addressed without the need for an intricate cable-transmission or reliance on software-based kinematic constraints. To the best knowledge of the authors, only six of such 2-DoF planar RCM mechanisms have been reported in detail among available literature (Table 1). What follows is a more detailed description of each.

Gijbels et al. proposed a mechanism addressing the concerns related to 1-DoF RCM mechanisms and 70 their reliance on a proximal means for a translational DoF 25. The mechanism combines 11 rigid bodies and a base, relying on a total of 16 rotative and 1 translational DoF. At the time of writing, the mechanism has been the kinematic basis for the development of two surgical robotic systems, and their application within vitreoretinal surgery during several in-vivo animal studies [26, 27] and a first-in-human clinical study 24 .

75 In a related follow-up work to 25, a framework for a class of 2-DoF planar RCM mechanisms with a translational DoF implemented distal from the end-effector is identified 28. While not explicitly dealing with the synthesis of a single mechanism, it introduces a generalized set of design rules to derive 2-DoF planar RCM mechanisms based on virtual parallelograms 29. This work was followed by a general framework by Long et al. 30] for the derivation of 2-DoF planar RCM mechanisms relying on pantograph mechanisms.

80 By means of demonstration, it concludes by synthesising a mechanism as prior detailed in both [25] and [28]. Based on the conducted literature review in this study the mechanisms as reported in [25], and later also proposed in [30, 23, remain among the least complex designs in terms of the number of linkages, joint locations, and the total number of joints.

Li et al. proposed a concept incorporating a passive prismatic joint at the proximal end of the mechanism 85 31. The mechanism combines 11 rigid bodies and a base, relying on a total of 17 rotative and 1 translational DoF.While it succeeds in removing the need for a translational drive at the end-effector, it requires a combined prismatic-rotative joint at the end-effector. Due to this, the previously described concerns of volume and mass would seem to be only partially mitigated. Furthermore, the presence of such a joint limits end-effector design and instrument compatibility.

Nisar et al. proposed an alternative mechanism in [32. Here, the presence of a downward protruding link is addressed, which can be considered as undesirable for surgical robotics as it moves in the direction of the patient. As such, it may lead to harmful contact if not mitigated, with for instance a cover, and may limit the safe placement of the mechanism in close vicinity of the patient. The mechanism combines 13 rigid bodies and a base, relying on a total of 19 rotative and 1 translational DoF. While still containing a downward link, it remains contained within the planar mechanism via the addition of a nested parallelogram, as such preventing it from protruding past the base. 


\begin{tabular}{|c|c|c|c|c|c|c|c|}
\hline Reference & [25] & [28, 30] & 31 & $32]$ & $33]$ & 34 & $*$ \\
\hline No. of linkages & 11 & 9 & 11 & 13 & 19 & 13 & 9 \\
\hline No. of joints & $16 \mathrm{R}, 1 \mathrm{~T}$ & $14 \mathrm{R}, 1 \mathrm{~T}$ & $17 \mathrm{R}, 1 \mathrm{~T}$ & $19 \mathrm{R}, 1 \mathrm{~T}$ & $28 \mathrm{R}, 3 \mathrm{~T}$ & $16 \mathrm{R}, 1 \mathrm{~T}$ & $14 \mathrm{R}, 1 \mathrm{~T}$ \\
\hline $\begin{array}{l}\text { Actuation on } \\
\text { base possible? }\end{array}$ & yes & yes & no & yes & yes & no & yes \\
\hline $\begin{array}{l}\text { Joints required on } \\
\text { end-effector axis? }\end{array}$ & $\begin{array}{l}\text { yes, } \\
2 \mathrm{R}\end{array}$ & $\begin{array}{l}\text { yes, } \\
2 \mathrm{R}\end{array}$ & $\begin{array}{l}\text { yes, } \\
2 \mathrm{R}, 1 \mathrm{~T}\end{array}$ & $\begin{array}{l}\text { yes, } \\
2 \mathrm{R}\end{array}$ & $\begin{array}{l}\text { yes, } \\
2 \mathrm{R}, 1 \mathrm{~T}\end{array}$ & $\begin{array}{l}\text { yes, } \\
1 \mathrm{~T}\end{array}$ & no \\
\hline
\end{tabular}

Table 1: Comparison of synthesised 2-DoF planar RCM mechanisms in literature. *Proposed in this work.

In a follow-up work by the same authors, an alternative mechanism was proposed which focussed on reducing the footprint of the planar mechanism [33. The mechanism combines 19 rigid bodies and a base, relying on a total of 28 rotative and 3 translational DoF. While the design indeed provides a small footprint at

100 the base, it does so by increasing mechanism complexity. Furthermore, similar to [31], a combined prismaticrotative joint at the end-effector becomes a requirement. This gives rise to the previously mentioned negative aspects such as additional end-effector volume and mass, limited space in the surgical scene, risk of patient contact, etc. At the time of writing, no physical demonstrators have been reported based on 32, 33, and a first full-scale prototype said to be under development. Given the larger number of rigid bodies and joints, key performance factors such as friction, play, stiffness and inertia could pose limitations. Therefore, it remains an open question whether in practice the added complexity and accompanying costs are a valid trade-off for their intended benefits.

Chen et al. proposed a design which relies on two double parallelogram structures present in two parallel planes, which are combined with a Peaucillier-Lipkin straight-line cell at the end-effector [34]. The mechanism combines 13 rigid bodies and a base, relying on a total of 16 rotative and 1 translational DoF. This combination enables a coupled rotation and translation at an RCM which can be actuated at the base of the two double parallelogram structures. A key advantage of the mechanism is its straightforward kinematics, which leads to a concise expression of the differential kinematic containing only a limited amount of trigonometric functions. However, it does so at the cost of introducing a large number of joints and linkages at the end-effector, more specifically a complete Peaucillier-Lipkin straight-line cell containing 7 rigid bodies, 8 rotative joints and one prismatic joint. Within the identified state-of-the-art, it arguably yields the most complex and voluminous end-effector.

Based on the literature review, it is clear that 2-DoF coupled RCM mechanisms are able to reduce the required mass and complexity by removing the need for actuation of the translational RCM DoF at the end-effector. However, all identified mechanisms share a remaining key design limitation, more specifically their reliance on several joints coinciding with or in near vicinity of the end-effector axis and surgical instrument. This limitation continues to pose limitations on the real-world application of these mechanisms during robot-assisted surgery. This concern is briefly mentioned in [28, which deals with a framework for deriving 2-DoF planar RCM mechanisms and is demonstrated by conceptually proposing three other kinematic embodiments. One of which enabling the omission of joints at the end-effector, which will form the foundation for the mechanism proposed and further synthesised in this work. This is further detailed in section II.

\subsection{Optimal kinematic design of parallel manipulators}

Kinematic design of parallel mechanisms involves the selection of a multitude of, mostly coupled, design parameters causing the design process to be non-trivial. As such, numerical methods are often used to determine acceptable or optimal kinematic designs [35]. Various approaches have been reported, during which a specific performance aspect of a mechanism is optimised. Lou et al. reported on an optimal kinematic design of a parallel manipulator aimed at optimizing workspace coverage 36. Nisar et al. described the optimisation of a 2-DoF planar RCM mechanism based on isotropic manipulability [33]. 
Selecting which parameters to optimize depends on the specific requirements of the use-case, and can involve optimizing a combination of multiple parameters. For instance, Lum et al. reported on an optimal kinematic design of a spherical mechanism for MIS during which both workspace coverage and isotropic manipulability were optimized [37.

\subsection{Objectives}

Even though the mechanism design initially described in 25] has been successfully used in its targeted application, several opportunities remain for improvement. Firstly, as is the case for all identified 2-DoF planar RCM mechanisms, it requires at least two rotative joints that intersect with the end-effector axis. Due to this, a limited amount of hinder-some volume and mass remains at the proximal end of the mechanism. Perhaps more importantly, this poses unnecessary challenges on the design of an end-effector and of a sterile-interface. This, in turn, causes compatibility with existing surgical instruments to be more difficult to realize. Second, it uses a downward protruding link, which may lead to harmful contact with the patient if not properly mitigated. As a result, one may be forced to resort to an overly long front parallelogram as well as a large surrounding base bracket in their design. This tends to reduce the overall stiffness, increase the system's footprint, and limit the placement of the mechanism in close-vicinity to the patient. Finally, the current design was configured heuristically using a limited set of design parameters, aimed primarily at providing workspace coverage for the central retinal area. While sufficient for its originally intended application, enabling broader usage within eye-surgery would be beneficial. Furthermore, the impact of a numerical optimization method applied on a similar, yet more generalized mechanism remains unknown.

This work is aimed at addressing the above concerns. Through the further adaptation of one the conceptual embodiments proposed in [28, a 2-DoF planar RCM mechanism is presented and analysed. Its improvements reside primarily in the advantageous reconfiguration of linkage geometries, providing a more generalized and potent set of kinematic expressions for the purpose of kinematic design. In addition, minor changes are made to the topology by decoupling previously combined joints locations, enabling further design freedom. Furthermore, a general numerical method is proposed to identify an optimal kinematic design for the proposed mechanism, and is implemented for the use-case of vitreoretinal surgery. Finally, in order to evaluate performance for the given use-case, a comparison with the existing mechanism is made.

\subsection{Structure}

The remainder of this work is structured as follows: A general description of the proposed mechanism, as well as the derivation of the inverse kinematics, forward kinematics, differential kinematics, and singularities, are provided in section 2 . The optimal design of the mechanism for the use-case of vitreoretinal microsurgery is detailed in section 3. The performance of the designed mechanism and its comparison with the prior design in section 4. Finally, a discussion of the presented work is provided in section 5, followed by a general conclusion.

\section{Mechanism description}

\subsection{Overview and construction}

Fig. 2 illustrates the application of the conceptual framework as proposed in 28. One side of a parallelogram is combined with a $1 \mathrm{R} 1 \mathrm{~T}$ joint fixated at a base. Upon the condition that the other side of the parallelogram is maintained parallel to the base, the motion of the $1 \mathrm{R} 1 \mathrm{~T}$ joint is copied from the $1 \mathrm{R} 1 \mathrm{~T}$ joint to a fixed location in space, thus creating a 1R1T RCM. Fig. $2 \mathrm{~b}$ shows an example of such a solution, being a rear dual parallelogram. This solution is deemed advantageous for its compactness, stiffness, and ability to implement passive gravity compensation using counterbalance weights. It should be noted that this configuration can be expanded with two additional rear linkages, thus enabling actuation of both parallelograms at the base. What follows remains relevant to both concepts.

Fig. 2p illustrates one of the conceptual embodiment as proposed in 28, where one additional design freedom is defined in the form of a parallel offset in two opposing parallelogram linkages in order to avoid 
coincidence of any joints with the end-effector axis. Such an offset has been previously identified [38 and advantageously implemented in RCM mechanisms for robot-assisted MIS [14].

In this work, three additional design freedoms are defined which are deemed advantageous for a real-world application of such a mechanism. Firstly, Fig. 2d shows that a parallel angular offset can be implemented, enabling a larger design space for end-effector design and a more advantageous configuration of the front parallelogram to the workspace. Secondly, Fig. 2e shows that the base of the rear copying mechanism can be offset from the axis between the LCM and the RCM. Thirdly, Fig. 2: shows that the 1R1T joint can be advantageously decoupled, thus omitting the need for a downward protruding link. It contains four additional design freedoms with respect to (w.r.t.) preceding designs, which are deemed advantageous for application in robot-assisted surgery. What follows is a more detailed description of this mechanism, along with a description of its kinematics.
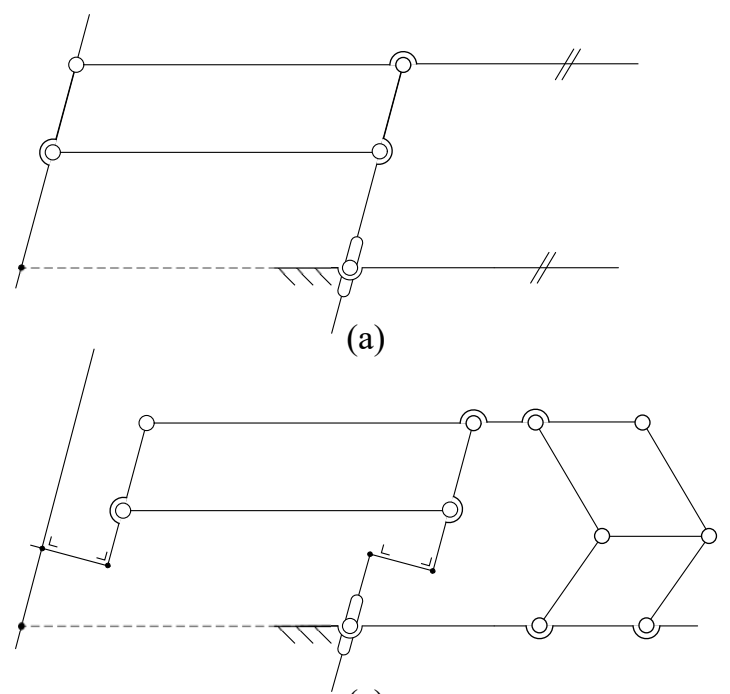

(c)

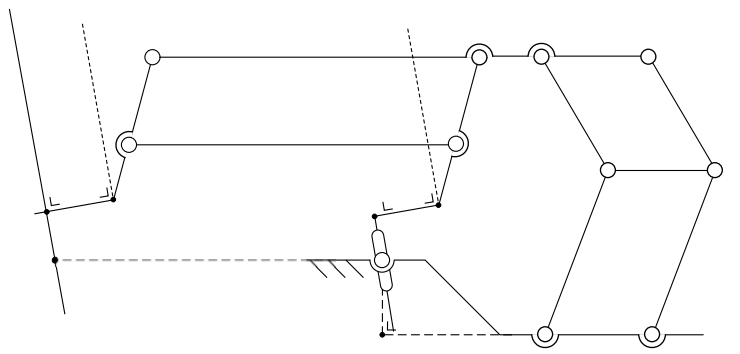

(e)

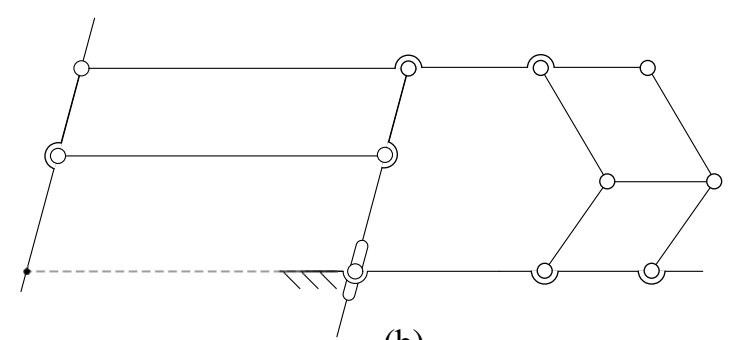

(b)

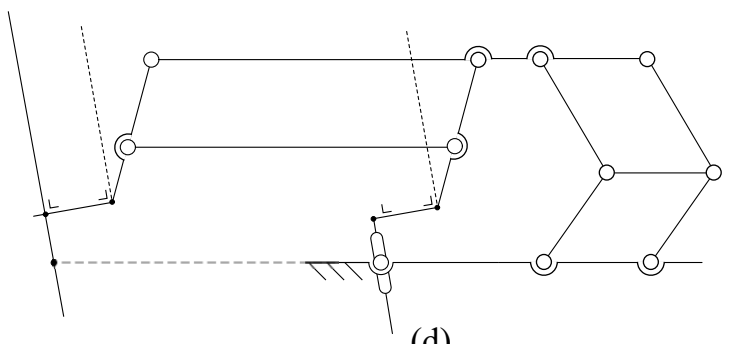

(d)

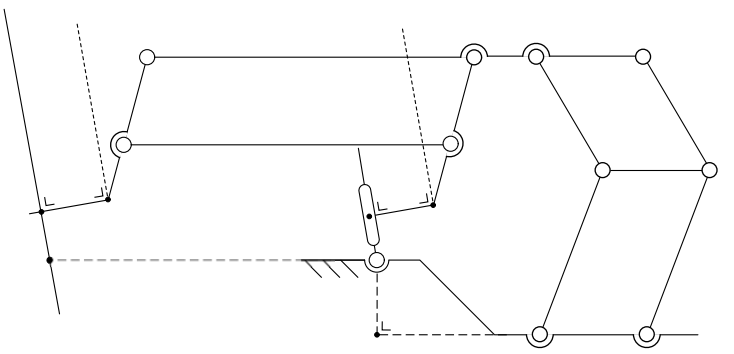

(f)

Figure 2: Construction of the proposed mechanism. It is readily understood from (a) that, while a rear dual parallelogram is implemented from (b) onwards, any means for maintaining parallelism at the rear is conceptually valid.

\subsection{Detailed description}

Fig. 3 provides an annotated illustration of the proposed mechanism. The 2-DoF planar RCM mechanism combines 9 rigid bodies with rigid linkage $H M L$ acting as a base, relying on a total of 14 rotative and 1 translational DoF implemented at 10 physical joint locations. Locations of interest are indicated with capital letters, more specifically rotative joints $B, C, D, E, F, H, I, J, K, L, M, N$, prismatic joint $G$, locations on rigid bodies $T, A, F$, and virtual points $H^{*}$ and $O$. The latter being the origin, and the location of the RCM. The RCM DoF are expressed at the instrument tip and are indicated as $R, \theta, \phi$, and $\psi$. Joint DoF are expressed as $q_{1} \ldots q_{4}$. Additional expressions for the 2-DoF planar kinematics are provided being 


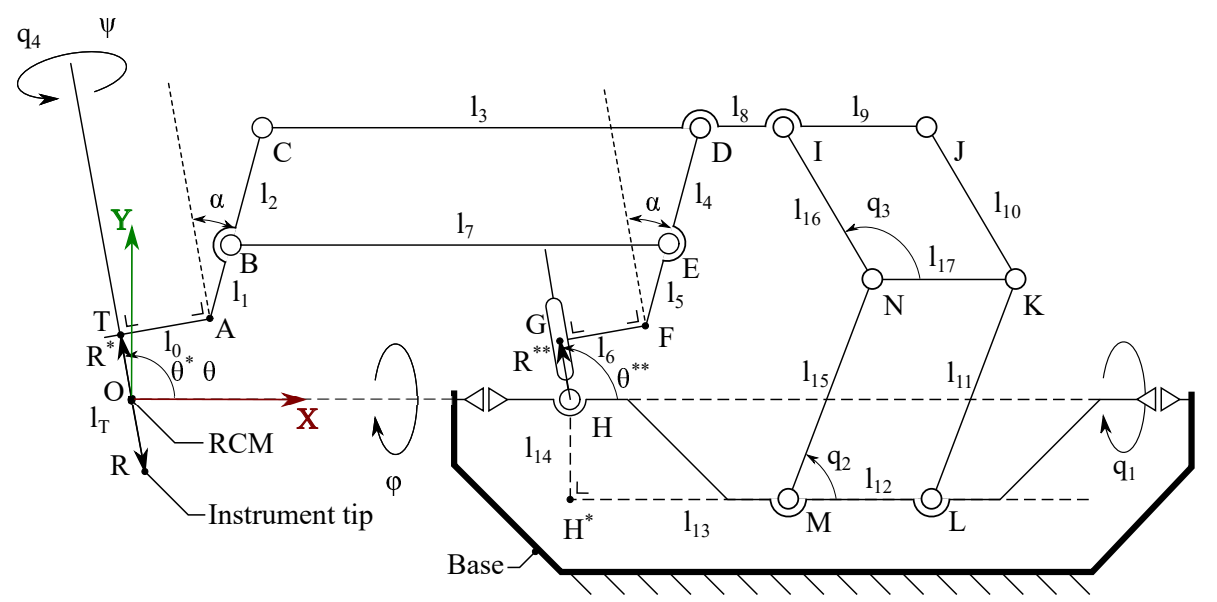

Figure 3: Annotated overview of the mechanism kinematics.

$R^{*}, \theta^{*}$ between the RCM and instrument fixation point $T$ as well as $R^{*} *, \theta^{*} *$ between locations $H$ and $G$. Mechanism configuration parameters are indicated as lenghts $l_{1} \ldots l_{17}$ and angle $\alpha$. Additionally, instrument length $l_{T}$ is defined between the tip and the fixation point $T$.

The four RCM DoF $R, \theta, \phi$, and $\psi$ defined at the tip of the instrument are realized via a combination of a 2-DoF planar RCM mechanism with two 1-DoF revolute joints. The functioning of the 2-DoF planar mechanism, which provides $\mathrm{RCM} \operatorname{DoF} R$ and $\theta$, can be understood as follows. A local center of motion is created through and about joint location $\mathrm{H}$ via the combination of a prismatic and a rotary joint. The movable part of this prismatic joint is fixated to rigid link GFED at location G, which is expressed as distance $R^{* *}$ and angle $\theta^{* *}$ w.r.t. joint location $\mathrm{H}$ and the $\mathrm{X}$-axis. More specifically, rigid link GFED is composed as follows. A straight link GF is fixated perpendicular to the translational direction of the prismatic joint at location G. GF is rigidly connected to straight link FED at location F, with the angle between GF and FED being $\frac{\pi}{2}+\alpha$. At locations $\mathrm{E}$ and $\mathrm{D}$ a rotational joint connects rigid link GFED to rigid links BE and CDIJ respectively. These three rigid links form a parallelogram BCDE with rigid link TABC, which shares an identical geometry with rigid link GFED. In turn, analogous to how rigid link GFED is fixated to the prismatic joint at location $\mathrm{G}$, rigid link TABC holds the instrument at location $\mathrm{T}$. The coordinates of $\mathrm{T}$ are expressed as $R^{*}$ and $\theta^{*}$ w.r.t. the origin and the X-axis. An RCM is created at the origin through and about which the instrument moves, provided that rigid link CDIJ remains parallel to the X-axis. This is achieved via two stacked parallelograms IJNK and NKML, which connect CDIJ with rigid link HML. As such, ML is by design parallel to the $\mathrm{X}$-axis. Indeed, $R^{* *}$ and $\theta^{* *}$ are equal to $R^{*}$ and $\theta^{*}$ respectively under the condition of the following equalities:

$$
\begin{aligned}
& l_{0}=l_{6} \quad l_{1}=l_{5} \\
& l_{9}=l_{17}=l_{12} \quad l_{10}=l_{16} \\
& \begin{aligned}
l_{2} & =l_{4} & l_{3}=l_{7} \\
l_{11} & =l_{15} &
\end{aligned}
\end{aligned}
$$

Consequently, $R^{* *}$ and $\theta^{* *}$ can then be expressed as RCM DoF $R$ and $\theta$ at the instrument tip by taking into account instrument length $l_{T}$. In addition to $R$ and $\theta$ provided by the 2-DoF planar mechanism, the third RCM DoF $\phi$ is implemented as 1-DoF revolute joint, of which the axis is collinear with the X-axis. As such, the planar mechanism rotates about the X-axis at both ends of rigid link HML. Finally, the remaining RCM DoF $\psi$ can be implemented as an additional 1-DoF revolute joint about the instrument's longitudinal axis at instrument fixation point $\mathrm{T}$.

Joint angles $q_{1}$ and $q_{4}$ are implemented by two independent 1-DoF revolute joints implemented in series with the 2-DoF planar RCM mechanism. As such, it can be readily understood that these are simply equal to angles $\phi$ and $\psi$ respectively. Therefore, what remains to be synthesized is the 2-DoF planar RCM mechanism, more specifically the correlations between joint angles $q_{2}, q_{3}$ and end-effector variables $R, \theta$. The analysis of both the inverse and forward kinematics is done with the aid of Fig. 3 . 
It should be noted that for the purpose of this study the choice for planar actuation of angles $q_{2}$ and $q_{3}$ is maintained from [25] as to enable a performance comparison related only to the kinematic architecture changes. The mechanism may also be driven via a combination of $\theta^{* *}$ and $q_{2}$ or a combination of $\theta^{* *}$ and $R^{* *}$. It is readily understood that former advantageously maintains the actuation at the base while avoiding two extra linkages, and that the latter advantageously simplifies the differential kinematics to four one-to-one relationships.

\subsection{Inverse kinematics}

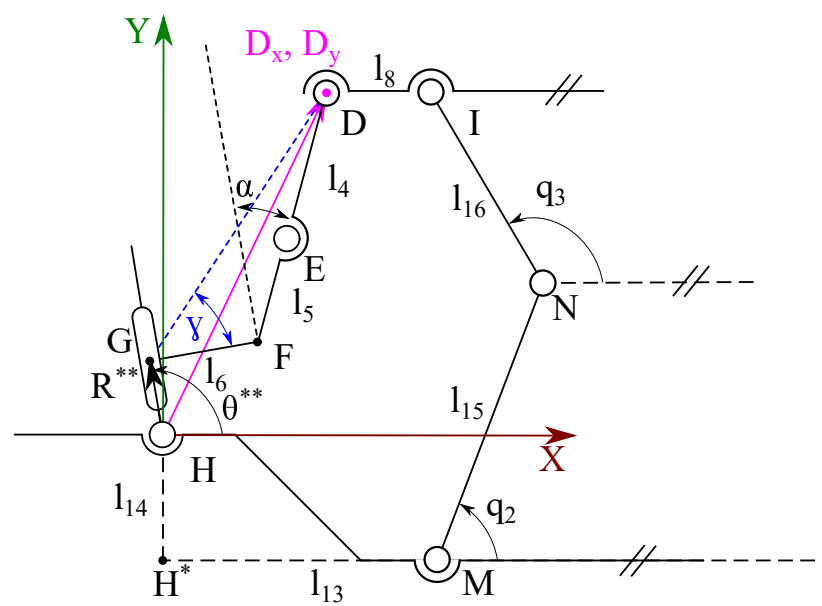

Figure 4: Annotated detail of planar loop HGFEDINM

The inverse kinematics describe the mechanism configuration as a function of end-effector variables $(R$, $\theta, \phi, \psi)$. The resulting mechanism configuration is fully described by the joint angles $\left(q_{1}, q_{2}, q_{3}, q_{4}\right)$.

$$
\left(q_{1}, q_{2}, q_{3}, q_{4}\right)=\mathcal{I} \mathcal{K}(R, \theta, \phi, \psi)
$$

$R$ and $\theta$ can be expressed in function of $R^{* *}$ and $\theta^{* *}$ as follows:

$$
\begin{aligned}
R & =R^{* *}-l_{T} \\
\theta & =\theta^{* *}
\end{aligned}
$$

Expressing the planar coordinates of $D$ w.r.t. $H$ in function of $R^{* *}$ and $\theta^{* *}$ gives:

$$
\begin{aligned}
& \mathbf{D}_{x}=R^{* *} \cos \left(\theta^{* *}\right)+l_{6} \sin \left(\theta^{* *}\right)+\left(l_{4}+l_{5}\right) \cos \left(\theta^{* *}-\alpha\right) \\
& \mathbf{D}_{y}=R^{* *} \sin \left(\theta^{* *}\right)-l_{6} \cos \left(\theta^{* *}\right)+\left(l_{4}+l_{5}\right) \sin \left(\theta^{* *}-\alpha\right)
\end{aligned}
$$

Expressing the same planar coordinates in function of $q_{2}$ and $q_{3}$ gives:

$$
\begin{aligned}
& \mathbf{D}_{x}=l_{15} \cos \left(q_{2}\right)+l_{16} \cos \left(q_{3}\right)+l_{13}-l_{8} \\
& \mathbf{D}_{y}=l_{15} \sin \left(q_{2}\right)+l_{16} \sin \left(q_{3}\right)-l_{14}
\end{aligned}
$$

From Eq. 4-7 the inverse kinematics as defined in Eq. 1 can be derived:

$$
\begin{aligned}
& q_{1}=\phi \\
& q_{2}=\operatorname{atan} 2\left(\frac{\mathbf{D}_{y}}{\mathbf{D}_{x}}\right) \pm \operatorname{acos}\left(\frac{\mathbf{D}_{x}^{2}+\mathbf{D}_{y}^{2}+l_{15}^{2}-l_{16}^{2}}{2 l_{15} \sqrt{\mathbf{D}_{x}^{2}+\mathbf{D}_{y}^{2}}}\right) \\
& q_{3}=\mp \operatorname{acos}\left(\frac{\mathbf{D}_{x}^{2}+\mathbf{D}_{y}^{2}-l_{15}^{2}-l_{16}^{2}}{2 l_{15} l_{16}}\right)+q_{2} \\
& q_{4}=\psi
\end{aligned}
$$


with $\mathbf{D}_{x}$ and $\mathbf{D}_{y}$ written in function of $R^{* *}$ and $\theta^{* *}$, which in turn can be expressed in function of $R$ and $\theta$ using equations 2 - 3 .

\subsection{Forward kinematics}

The forward kinematics describe the mechanism configuration as a function of the joint angles $\left(q_{1}, q_{2}\right.$, $\left.q_{3}, q_{4}\right)$. The resulting mechanism configuration is fully described by the end-effector variables $(R, \theta, \phi, \psi)$.

$$
(R, \theta, \phi, \psi)=\mathcal{F} \mathcal{K}\left(q_{1}, q_{2}, q_{3}, q_{4}\right)
$$

A pose-independent triangle can be formed between the points $D, F$, and $G$ (Fig. 4). Using the cosine rule, the triangle edge $|D G|$ is found by:

$$
|D G|=\sqrt{\left(l_{4}+l_{5}\right)^{2}+l_{6}^{2}-2\left(l_{4}+l_{5}\right) l_{6} \cos (\pi / 2+\alpha)}
$$

The angle $\gamma$ between edges $|F G|$ and $|D G|$ of triangle $D F G$ can subsequently be determined by once again applying a cosine rule:

$$
\gamma=\operatorname{acos}\left(\frac{|D G|^{2}+l_{6}^{2}-\left(l_{4}+l_{5}\right)^{2}}{2 l_{6}|D G|}\right)
$$

With $|D G|$ and $\gamma$ known, triangle $D G H$ can be formed. Using the cosine rule, the triangle edge $|D H|$ is found by:

$$
|D H|^{2}=|D G|^{2}+R^{* * 2}-2|D G| R^{* *} \cos (\gamma+\pi / 2)
$$

Which can be rewritten as a polynomial second order equation in function of $R^{* *}$ as:

$$
R^{* * 2}-2 \cdot|D G| \cos (\gamma+\pi / 2) R^{* *}+|D G|^{2}-|D H|^{2}=0
$$

Solutions of Eq. 16 are then found as:

$$
R_{1,2}^{* *}=|D G| \cos (\gamma+\pi / 2) \pm \sqrt{|D G|^{2} \cos ^{2}(\gamma+\pi / 2)-|D G|^{2}+|D H|^{2}}
$$

Once $R^{* *}$ is known, $\theta^{* *}$ can be determined by combining the angle between the base and $H D$ with the angle between edges $|H G|$ and $|H D|$ of triangle $D G H$.

$$
\theta_{1,2}^{* *}=\operatorname{atan} 2\left(\frac{\mathbf{D}_{y}}{\mathbf{D}_{x}}\right) \pm \operatorname{acos}\left(\frac{|D H|^{2}+R^{* * 2}-|D G|^{2}}{2 R^{* *}|D H|}\right)
$$

From Eq. 17 and 18 the forward kinematics as defined in Eq. 12 can be derived:

$$
\begin{aligned}
& \phi=q_{1} \\
& R=|D G| \cos (\gamma+\pi / 2)+\sqrt{|D G|^{2} \cdot \cos ^{2}(\gamma+\pi / 2)-|D G|^{2}+|D H|^{2}}-l_{T} \\
& \theta=\operatorname{atan} 2\left(\frac{\mathbf{D}_{y}}{\mathbf{D}_{x}}\right)+\operatorname{acos}\left(\frac{|D H|^{2}+R^{* * 2}-|D G|^{2}}{2 R^{* *}|D H|}\right) \\
& \psi=q_{4}
\end{aligned}
$$




\subsection{Differential kinematics}

The differential kinematics describes the relationship between the joint velocities $\dot{q}$ and end-effector velocities $\dot{x}$. For the given 4 -DoF mechanism, this results in a 4 x4 matrix $J$ with each cell containing a linear coefficient found by taken the partial derivative of a given end-effector positional expression with respect to the related joint angle.

$$
\begin{gathered}
\dot{x}=J \dot{q} \\
J_{i, j}=\frac{\partial x_{i}}{\partial q_{j}} \\
{\left[\begin{array}{c}
\dot{R} \\
\dot{\theta} \\
\dot{\phi} \\
\dot{\psi}
\end{array}\right]=\left[\begin{array}{cccc}
0 & J_{1,2} & J_{1,3} & 0 \\
0 & J_{2,2} & J_{2,3} & 0 \\
1 & 0 & 0 & 0 \\
0 & 0 & 0 & 1
\end{array}\right]\left[\begin{array}{c}
\dot{q}_{1} \\
\dot{q}_{2} \\
\dot{q}_{3} \\
\dot{q}_{4}
\end{array}\right]}
\end{gathered}
$$

\subsection{Singularity analysis}

Singular configurations of the mechanism are derived heuristically by means of geometric analysis for the available movement range of the mechanism. A total of 8 singularities are identified. Each physical parallelogram yields two singular positions, occurring during the collinear alignment of all four linkages. This renders a total of 6 singular positions for the proposed mechanism, occurring when parallelogram angles $q_{2}, q_{3}$, or $\zeta$ are set at either 0 or $\pi$. In addition, two additional singularities exist in the dual rear parallelogram during the collinear alignment of two opposing parallelogram linkages, more specifically linkages $I N$ w.r.t. $N M$ and simultaneously $J K$ w.r.t. $K L$. These two singularities occur when the difference between $q_{2}$ and $q_{3}$ are set at either 0 or $\pi$.

$$
q_{2}=\{0, \pi\} \quad q_{3}=\{0, \pi\} \quad \zeta=\{0, \pi\} \quad\left(q_{3}-q_{2}\right)=\{0, \pi\}
$$

\section{Mechanism Optimization}

For the purpose of this paper, a design optimisation for the use-case of vitreoretinal surgery is detailed. Fig. 5 shows the previously described mechanism, with its RCM positioned at the incision site of an eyeball. Note that an additional design parameter $\beta$ is added, enabling the mechanism to be oriented w.r.t. the patient by rotating it at the RCM about the Z-orientation. Furthermore, based on the previously identified parameter equalities, the previously described RCM mechanism is redefined using only relevant design parameters, more specifically parameters $\alpha, \beta, l_{3}, l_{4}, l_{5}, l_{6}, l_{8}, l_{9}, l_{13}, l_{14}, l_{15}, l_{16}, l_{T}$. Selection of these parameters during mechanism design is non-trivial. Therefore, a numerical approach is proposed for at least an additional subset of these parameters, aimed at providing an optimal set of design parameters given a set of to-be-optimized performance metrics. What follows is a detailed description of the implemented optimal design algorithm, divided into three segments. First, the chosen optimal design metrics are detailed in subsection 3.1 for the use-case of robot-assisted vitreoretinal surgery. It is clear that a similar approach can be followed for other applications. Second, the chosen parameter ranges and mechanical constraints are defined in subsection 3.2. Third, a pseudocode description of the optimal design algorithm is detailed in subsection 3.3 .

\subsection{Metrics}

\subsubsection{Workspace coverage}

A desired workspace is identified, representing a foreseeable planar workspace during vitreoretinal surgery. cross-sections, as well as the mean anatomy, are approximated (Fig. 6). The resulting five cross-sections 


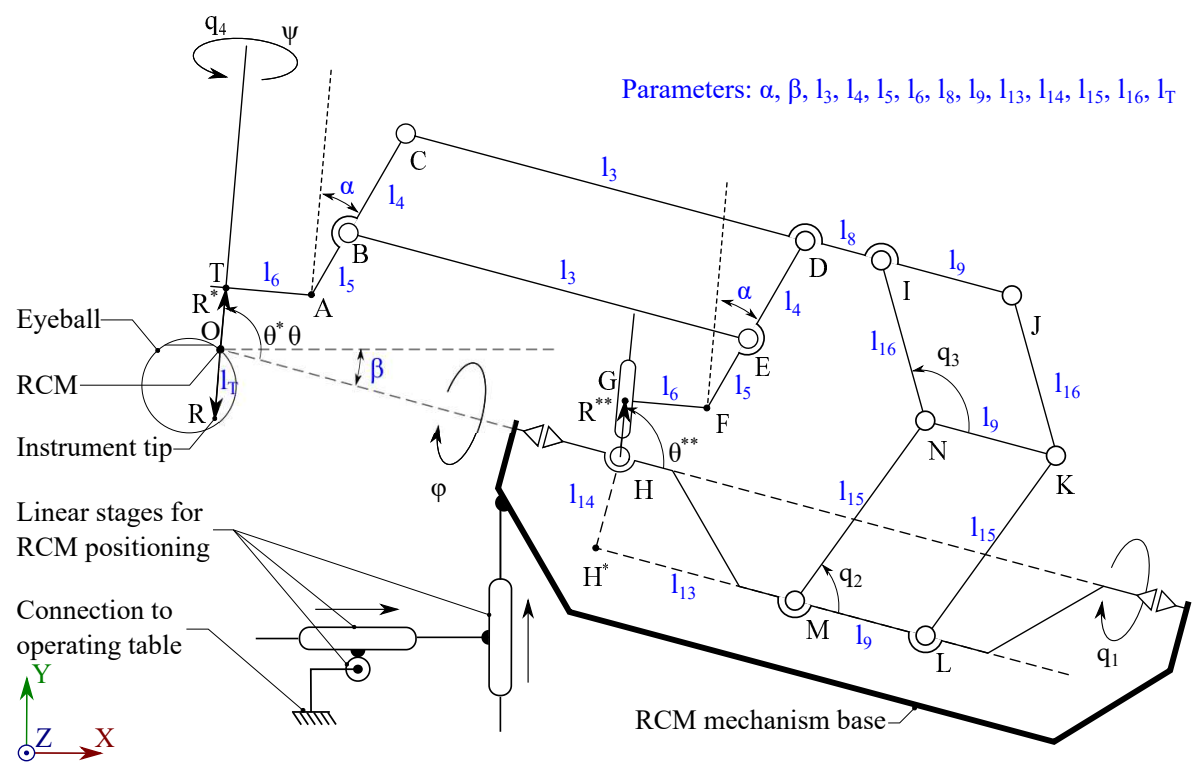

Figure 5: Annotated overview of the planar mechanism design parameters

are centred at their foreseeable surgical entry point. The central retinal area is defined as a 90 deg range centred at the backside of the eye. The additional extraocular area is defined to provide a safe tip position outside the eye during setup and instrument changes. Based on the feedback of cooperating surgeons, the intraocular area was limited to a fully horizontal instrument. From an application point of view, mechanism performance might be more important in critical areas of the workspace, and less important in others. For instance in eye surgery, one might consider the workspace area in near vicinity to the sensitive retina as more critical than the rest of the surgical workspace. To be able to factor in such considerations in this study, the overall desired workspace is divided into three areas of interest: extraocular, intraocular, and retinal (Fig. 6). These will be used to evaluate zone-specific performance.

For each possible mechanism design, the reachable workspace and related metrics will be evaluated as a point cloud based on a pre-defined range of joint positions. More specifically, the workspace of each mechanism design is computed using the forward kinematics and a predefined set of joint angles. This approach is considered advantageous, as this allows for all mechanical constraints declared in joint coordinates to be taken into account with a single computation prior to the algorithm. However, due to this approach, point cloud density will not be homogeneous across the workspace and will vary between different mechanism designs. This forms a concern when wanting to derive a single scalar metric to represent a workspace group of points in a given zone, as high-density areas will be overrepresented in the resulting scalar. In order to prevent this bias due to varying point density, the following approach is adopted. A point grid is created or each region of interest, filling each region with equidistant points. These point grids were then used to determine the nearest neighbour for each point in the mechanism workspace point cloud, through the application of a minimum distance threshold. As such, we are left with a homogeneous point cloud, unaffected by varying point density. The first set of scalar metrics based on this point grid is simply the degree to which the three areas of interest are covered by the workspace of a given kinematic design. These will be further referred to as covextraocular, $\operatorname{cov}_{\text {intraocular }}$, and $\operatorname{cov}_{\text {retinal }}$.

\subsubsection{Potential energy}

The mechanism should not move by itself under the influence of gravitational loads. This is the case when these loads are balanced adequately and remain below the static friction threshold of the mechanism. Compensation of gravitational loads to an acceptable level can be either achieved actively using controlled actuators or passively using elements such as springs or counterweights. In the case of a low friction 


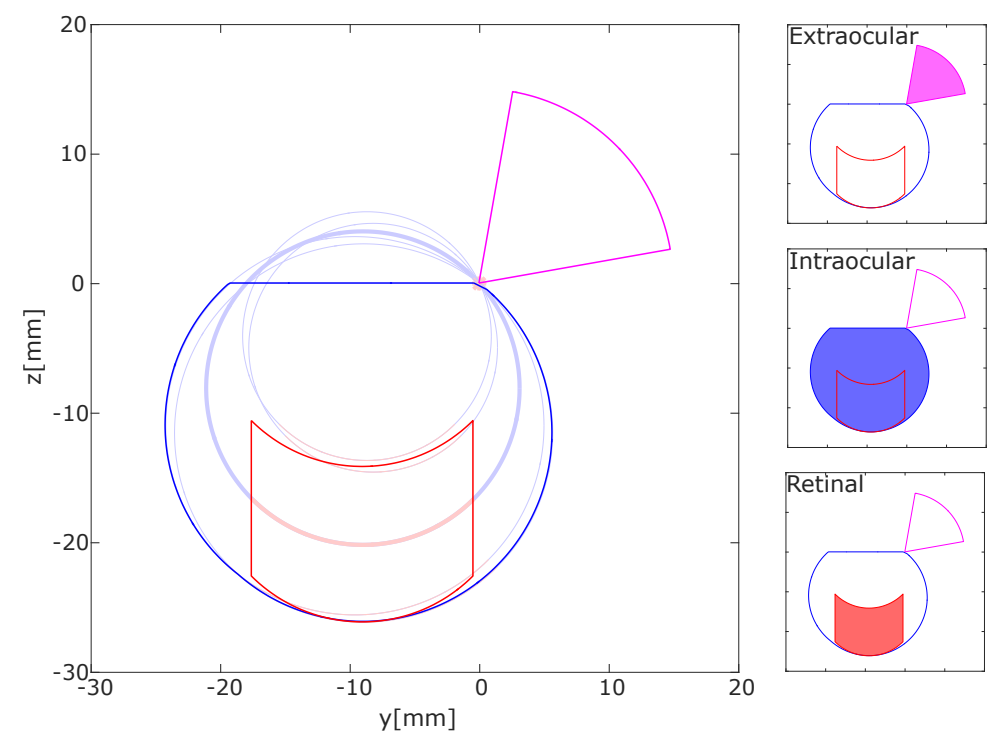

Figure 6: (Left) Desired planar workspace for vitreoretinal surgery based on 1st to 99th percentile anatomical variance for the human eye. (Right) An annotated overview of each defined area of interest within the planar workspace. More specifically: an extraocular, intraocular, and retinal area is defined and highlighted. These are used to identify area-specific performance metrics.

cooperative mechanism, such compensation needs to be designed with a low margin for error. Ideally, the user experiences minimal variation of gravitational handle forces during use. Given the above considerations, it is considered optimal to minimize both the average and the variance of the to be compensated amount of gravitational loads. This can be modelled by reviewing the potential energy term of the mechanism for all workspace points while making reasonable assumptions on linkage materials and cross-sections.

Given gravitational acceleration vector $\mathbf{g}$, the potential energy state of the mechanism with $n$ rigid bodies in a given joint configuration is determined by:

$$
\begin{aligned}
E_{p} & =\sum_{i=1}^{n} \mathbf{g}^{T} m_{i} C O M_{i} \\
m_{i} & =A l_{i} \rho_{i}
\end{aligned}
$$

where for link $i$ a mass $m_{i}$ is approximated by assuming a constant cross-section $A$ multiplied with linkage length $l_{i}$ and density $\rho_{i}$. Consequently, the link's centre of mass location $C O M_{i}$ to lie at $\frac{L_{i}}{2}$ within the link's local coordinate frame. After solving the forward kinematics, it is considered straightforward to evaluate the potential energy according to (27) and (28) for each given joint position. For the sake of brevity, the full expression of the potential energy function is therefore not detailed in this paper.

\subsubsection{Manipulability}

The mechanism will be used as a cooperatively-controlled robot of the impedance-type. Such can be considered as a system generating output forces in function of input velocities. Herein, the mechanism can be seen as a variable transmission between handle and joints for both velocities and forces, of which the transmission ratios vary across the workspace. As such, the ease with which the mechanism is able to cooperate in a given joint configuration can be reviewed by determining manipulability ellipsoids [39. Manipulability is reviewed in 3-DoF and is defined as the degree of isotropy of the generated handle forces given unity velocity inputs at the handle. Fully isotropic behaviour at a given point in the workspace would mean exactly the same force-velocity correlation in each direction. It should be noted that the RCM DoFs as currently defined combine rotations and translations. Combining rotational and translational units has 
been identified to limit the representative value of manipulability analysis and its eventual usage for optimal design [40. For this use-case, this concern is addressed as follows. Manipulability is computed at the handle - rather than the instrument tip or at the RCM - as this is where a user applies and experiences forces and velocities when interacting with the mechanism. As such, the three RCM DoFs can be reviewed as three translations applied by the user at a given point at the handle, allowing manipulability to be reviewed using DoF which all share the same unit. For the purpose of this paper, the handle is defined as the location of instrument fixation point T. An additional transmission matrix $\lambda$ is added for converting the translation and two rotations occurring at the RCM into three translations at the handle $\left(F_{h}=\lambda F_{x}\right)$. A scalar $l_{h}$ is used to indicate the fixed distance between the instrument tip and point $T$. For determining manipulability, joint impedance gain matrix $C_{q}$ is defined as an identity matrix, representing unit impedance gain at each joint. The manipulability eigenvector $M$ is determined as follows:

$$
\begin{gathered}
M=\operatorname{eig}\left(\lambda\left(J^{T}\right)^{-1} C_{q} J^{-1} \lambda^{T}\right) \\
\lambda=\left[\begin{array}{ccc}
1 & 0 & 0 \\
0 & 1 /\left(r+l_{h}\right) & 0 \\
0 & 0 & 1 /\left(r+l_{h}\right)
\end{array}\right]
\end{gathered}
$$

With $M$ being a $3 \times 1$ eigenvector representing an ellipsoid with three principal axes, of which axis length $r_{i}$ can be found by:

$$
r_{i}=\frac{1}{\sqrt{M_{i}}}
$$

In order to easily review the manipulability, the eigenvalue-based ellipsoid is reduced to a scalar $M_{\text {spher }}$ representing the ellipsoid's sphericity. The obtained value varies from zero to one with one indicating an ideal sphere, representing the highest degree of isotropy. For the purpose of this paper, it is assumed that a more isotropic manipulability is optimal and desired.

$$
M_{\text {spher }}=\operatorname{sphericity}(M)
$$

\subsubsection{Reflected joint stiffness}

During a cooperative positioning task, the robotic system and the user simultaneously grasp and position a surgical instrument to a desired location, after which the instrument position may be maintained using the joint actuators and position sensors to lock the mechanism. At this point, any change in forces occurring at the handle - including the user's hand release or grasp - will cause a positional error due to overall stiffness of the robot. The overall robot stiffness is influenced by the structural stiffness of the mechanism, as well as the stiffness of the joint-actuator transmission and the actuator itself. An initial analysis of the mechanism stiffness at the handle is included, by reviewing the reflected joint and transmission stiffness only. Similarly to manipulability, the Jacobian matrix can be used to determine reflected stiffness [35. The reflected stiffness matrix $K$ can be found by Eq. 33. with $K_{q}$ being a diagonal matrix containing the joint stiffness of each actuated joint. Note that as the current aim of this analysis is to compare different kinematic designs relative to each other, the currently selected value for actuated joint stiffness does not need to be representative, but merely kept consistent throughout the analysis. As such, joint stiffness is currently set to unity, which is to be interpreted as $1 \mathrm{~N} \mathrm{~mm} \mathrm{rad}^{-1}$ for each actuated joint. Generally, a higher value for reflected stiffness is assumed optimal and desired.

$$
K=\lambda\left(J^{T}\right)^{-1} K_{q} J^{-1} \lambda^{T}
$$

In order to simplify the analysis, the eigenvalues of the reflected stiffness matrix $K$ are determined, each representing a decoupled stiffness factor along its principal axis. In turn, these are reduced to a scalar $K_{\text {magn }}$ by computing their Euclidean norm. As such, representing the overall reflected stiffness magnitude in $3 \mathrm{DoF}$. For the given use-case, a kinematic design with a relatively higher reflected stiffness can be seen as advantageous. It is therefore assumed that a higher reflected stiffness value is optimal and desired. 


\subsection{Parameters and constraints}

\subsubsection{Parameters}

Mechanism parameters $\alpha, \beta, l_{4}, l_{5}$, and $l_{6}$ were chosen as constants based on the following two considerations: 1) avoiding front parallel singularities, 2) accommodating a hand holding a tool using a conventional grip. More specifically, the required angular range of the front parallelogram - expressed as $\zeta$ about joint B - to fully cover the desired workspace is approximated as $120^{\circ}$ (Fig. 7). In order to maximally avoid the singularities occurring at $\zeta=0^{\circ}$ and $\zeta=180^{\circ}$, zeta is to be $90^{\circ}$ when the instrument is at the middle of its required angular range. This occurs when $\beta-\alpha=30^{\circ}$ for the given workspace approximation. Furthermore, $l_{4}$ is set to a minimum of $30 \mathrm{~mm}$, enabling front parallelogram BCDE to close - approaching angular extremes of workspace - while maintaining a minimum clearance of $15 \mathrm{~mm}$ between linkages $C D$ and $B E$. Finally, $l_{5}, l_{6}$, and $\alpha$ are heuristically chosen such that a minimum of available space is provided for an instrument holder usable for handheld operation. Based on these considerations, the following constants were selected:

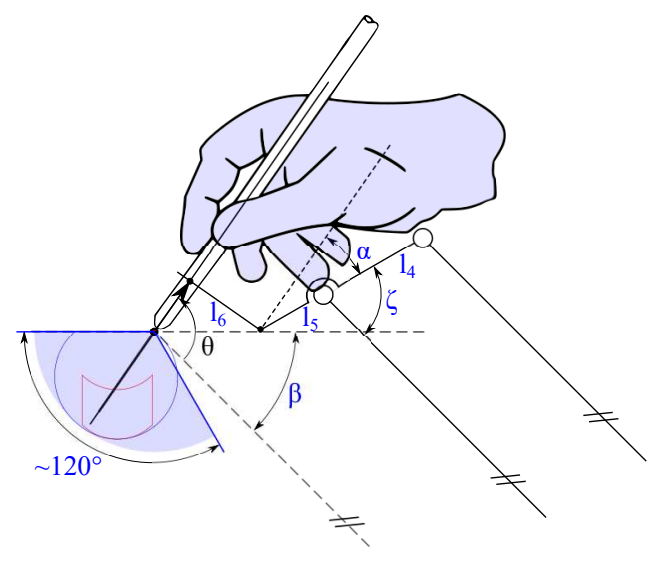

Figure 7: Front parallelogram parameters

$$
\alpha=15^{\circ} \quad \beta=45^{\circ} \quad l_{4}=30 \mathrm{~mm} \quad l_{5}=10 \mathrm{~mm} \quad l_{6}=40 \mathrm{~mm}
$$

It should be noted that while relevant in a later design phase such as clearance for patient anatomy and passive mechanism compliance, $l_{3}$ and $l_{9}$ do not affect the previously detailed performance metrics used in the current optimisation. For the purpose of this paper $l_{3}$ and $l_{9}$ are set to $160 \mathrm{~mm}$ and $75 \mathrm{~mm}$ respectively, which are similar to those used in a related design [24. The remaining mechanism parameters were chosen as discrete ranges within reasonable limits. The discretization step of each range was taken as $5 \mathrm{~mm}$, resulting in a combined total of 40824 to-be-reviewed kinematic designs within a 5-dimensional design space.

$$
\begin{aligned}
l_{15} & =50 \ldots 90 \mathrm{~mm} \\
l_{16} & =50 \ldots 90 \mathrm{~mm} \\
l_{13} & =20 \ldots 60 \mathrm{~mm} \\
l_{14} & =5 \ldots 40 \mathrm{~mm} \\
l t & =40 \ldots 70 \mathrm{~mm}
\end{aligned}
$$

\subsubsection{Kinematic constraints}

Kinematic constraints are applied at two instances throughout the algorithm. A first set of kinematic constraints is applied at joint level, where the operating range of each joint is identified. These are combined into a set of reachable joint configurations, based on kinematic joint constraints. In this case, preventing the parallelograms angles $q_{2}$ and $q_{3}$ from reaching their singularities at $0^{\circ}$ and $180^{\circ}$ as well as preventing rear dual parallelogram from reaching singularity poses at $q_{2}=q_{3}$ and $q_{2}=\pi+q_{3}$. A limit of $15^{\circ}$ is heuristically 
chosen as to-be-implemented mechanical joint limit for avoiding the singularity regions. As such, the set of permissible joint configurations is determined as follows:

$$
\begin{aligned}
& 15^{\circ} \leq q_{2} \leq 165^{\circ} \\
& 15^{\circ} \leq q_{3} \leq 165^{\circ} \\
& 15^{\circ} \leq\left(q_{3}-q_{2}\right) \leq 165^{\circ}
\end{aligned}
$$

An additional subset of kinematic constraints is applied after computing the forward kinematics. A rotational limit for $\theta^{* *}$ is defined, ensuring that the front parallelogram does not reach singularity positions at $(\theta+\alpha)=$ 0 and $(\theta+\alpha)=\pi$. Furthermore, an insertion limit for $R^{* *}$ is implemented, based on a foreseeable mechanical constraint of $30 \mathrm{~mm}$ due to the presence of a prismatic joint.

$$
\begin{aligned}
& 45^{\circ} \leq \theta^{* *} \leq 165^{\circ} \\
& R^{* *} \geq 30 \mathrm{~mm}
\end{aligned}
$$

\subsection{Numeric algorithm}

\subsubsection{Psuedocode}

A simple optimal design algorithm was developed and implemented for the purpose of this paper (Algorithm 1) .

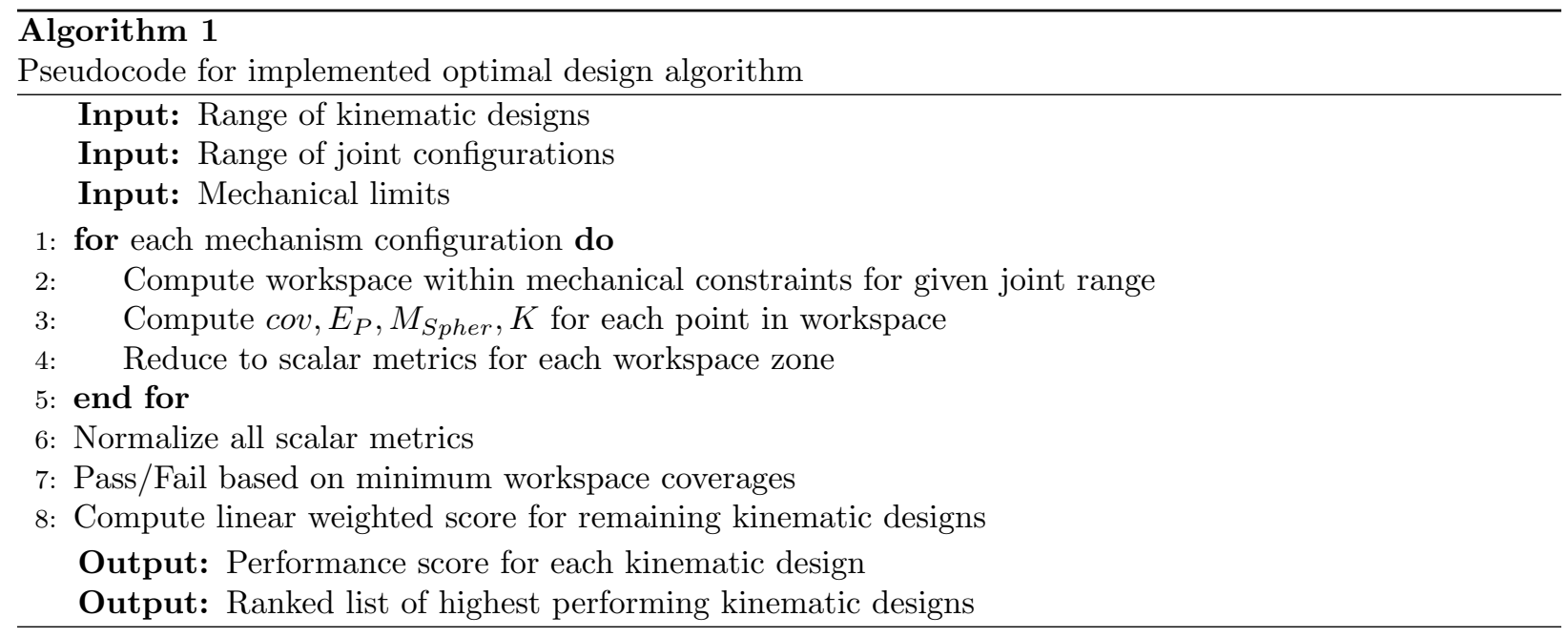


percentage of workspace coverage for each zone, being $99 \%, 75 \%$, and $50 \%$ for retinal, intraocular, and extraocular coverage respectively. It should be noted that while the retinal area represents a surgical workspace requirement, the defined intraocular and extraocular areas represent foreseeable areas of surgical utility. As such, their full coverage is desirable, but not needed for a minimum acceptable solution.

For the remaining minimum viable kinematic designs, a score function is applied to produce a ranking on which the final selection is based. High scores maximize intraocular workspace coverage, retinal workspace coverage, overall isotropic manipulability, and overall reflected stiffness, while minimizing overall variance of potential energy, variance of manipulability, and cumulative length of the rear parallel loop. Similar to a mean squared error cost function, the implemented score function Eq. 45 provides the mean sum of the squared score of each normalized metric. For the purpose of this analysis, all metrics are currently deemed equally important. As such, no individual weighting factors are included.

$$
\text { score }=\frac{1}{7}\left[\left(\operatorname{cov}_{\text {ret }}^{\prime}\right)^{2}+\left(\operatorname{cov}_{\text {intr }}^{\prime}\right)^{2}+\left(\operatorname{med}_{M}^{\prime}\right)^{2}+\left(\operatorname{med}_{K}^{\prime}\right)^{2}+\left(I Q R_{E_{p}}^{\prime}-1\right)^{2}+\left(I Q R_{M}^{\prime}-1\right)^{2}+\left(L_{R L}^{\prime}-1\right)^{2}\right]
$$

\section{Results}

\subsection{Algorithm}

The above-described algorithm was implemented in MATLAB R2018b (Mathworks, Inc., United States) and executed using a personal computer (Dell Precision 5530, I7-8850H, 32GB RAM), requiring approximately $8 \mathrm{~h}$ to complete. A total of 3794 out of 40824 possible kinematic designs provided at least the minimum required workspace coverage. This subset was subsequently ranked using the score function in order to select a final set of parameters. Fig. 8 depicts the scores of all minimum viable kinematic designs sorted from low to high. It can be noted that the approximately 500 highest scoring designs increasingly differentiate themselves, with the highest scoring design residing at a clear optimum. The highest scoring kinematic design is considered as the resulting optimal solution.

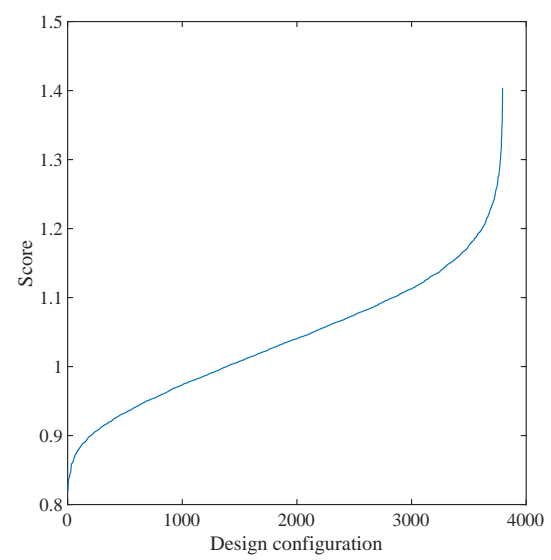

Figure 8: Score progression of the 3794 identified minimum viable kinematic designs, sorted from low to high.

\subsection{Configured mechanism and workspace}

The resulting mechanism parameters were identified as:

$$
\begin{aligned}
& l_{6}=40 \mathrm{~mm} \quad \alpha=15^{\circ} \quad \quad \beta=45^{\circ} \quad l_{5}=10 \mathrm{~mm} \quad l_{4}=30 \mathrm{~mm} \\
& l_{16}=50 \mathrm{~mm} \quad l_{15}=90 \mathrm{~mm} \quad l_{13}=50 \mathrm{~mm} \quad l_{14}=15 \mathrm{~mm} \quad l_{T}=60 \mathrm{~mm}
\end{aligned}
$$




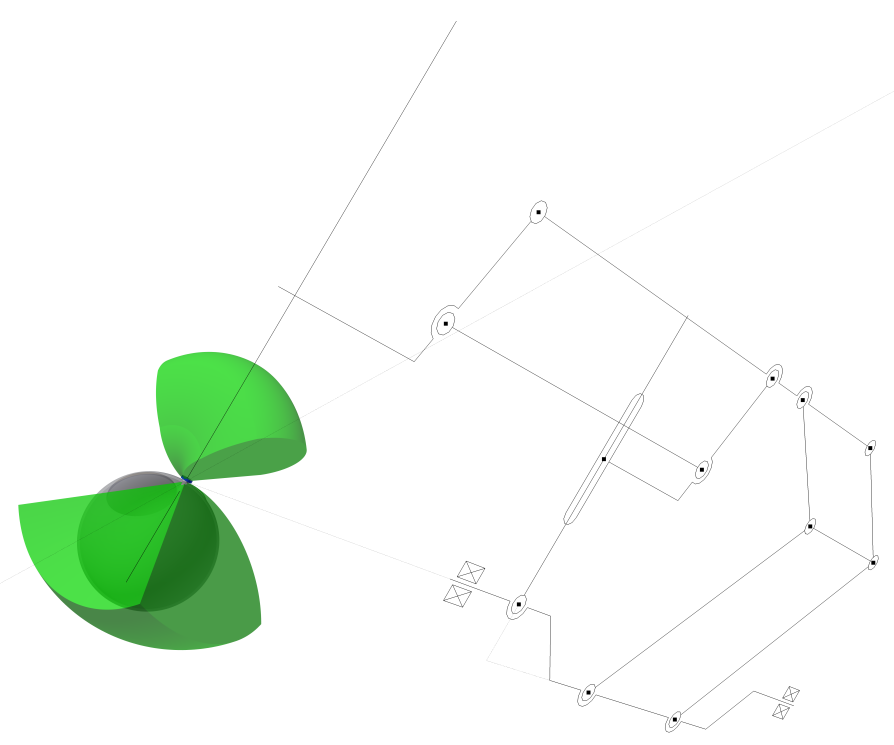

Figure 9: Resulting 3-DoF workspace and mechanism after optimisation.

The resulting planar mechanism is able to fully cover all foreseeable locations of the central retinal area, and is able to cover $93 \%$ of the foreseeable planar intraocular area. Fig 9 provides an illustration of the kinematic design and the resulting workspace volume when expanded with the additional rotational DoF $\phi$ for a range of $-45^{\circ} \ldots 45^{\circ}$. An eyeball with mean anatomy - outer diameter $24.2 \mathrm{~mm}$ - is added within the workspace envelope for providing a sense of scale.

\subsection{Metrics and comparison to prior mechanism}

\begin{tabular}{lcccc}
\hline & Prior & Proposed & Change [\%] & \\
\hline $\operatorname{cov}_{\text {intraocular }}[\%]$ & 56 & 93 & +66 & higher is better \\
$\operatorname{cov}_{\text {retina }}[\%]$ & 98 & 100 & +2 & higher is better \\
$\operatorname{med}_{M}[0 \ldots 1]$ & 0.97 & 0.94 & -3 & higher is better \\
$\operatorname{med}_{K}[N / \mathrm{mm}]$ & 0.07 & 0.05 & -29 & higher is better \\
$I Q R_{E p}[\mathrm{~J}]$ & 220.02 & 152.09 & -31 & lower is better \\
$I Q R_{M}[0 \ldots 1]$ & 0.05 & 0.03 & -40 & lower is better \\
\hline
\end{tabular}

Table 2: Comparison of selected performance metrics between the prior and newly proposed kinematic design. In order to provide an initial comparison w.r.t. the prior kinematic design, the same workspace analysis and related metrics were computed using the kinematics as reported in 25. Table 2 shows the comparison between selected performance metrics and shows the relative change along with a qualitative interpretation. It should be noted that for the purpose of this comparison, the values of the performance metrics shown are those before normalisation. When compared, some relevant differences in terms of workspace coverage can be noted. The intraocular workspace of the planar mechanism improves with $66 \%$ while providing coverage of the entire retinal zone. When reviewed in $3 \mathrm{DoF}$ using the same joint range for $q_{1}$, the resulting useful workspace volume increases nearly threefold. Before further comparing both mechanisms in terms of metrics, it should be noted that all of the following statements remain subject to change, as a final design and dimensioning phase is required. As such, a final prototype design could deviate - positively or negatively 


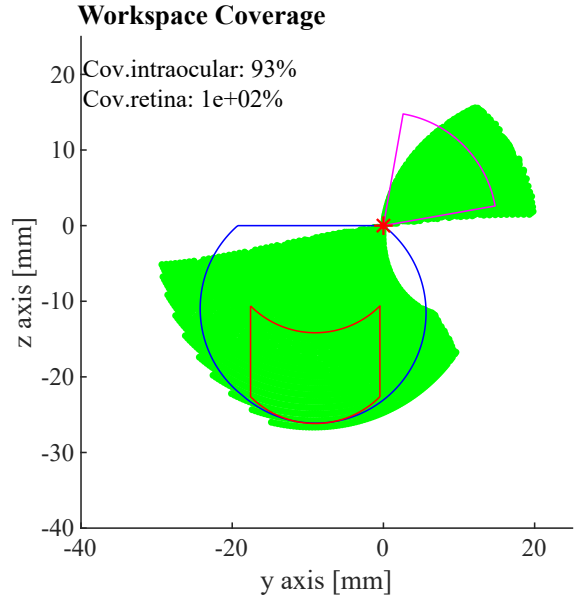

(a)

Potential Energy, planar [J]

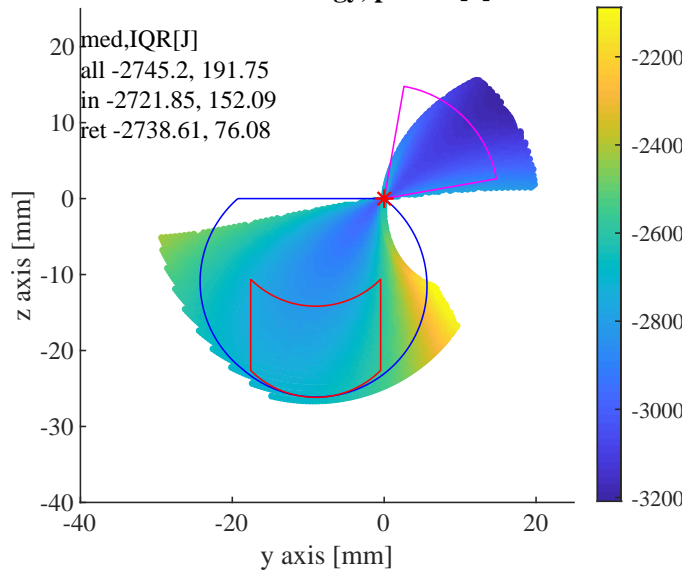

(c)

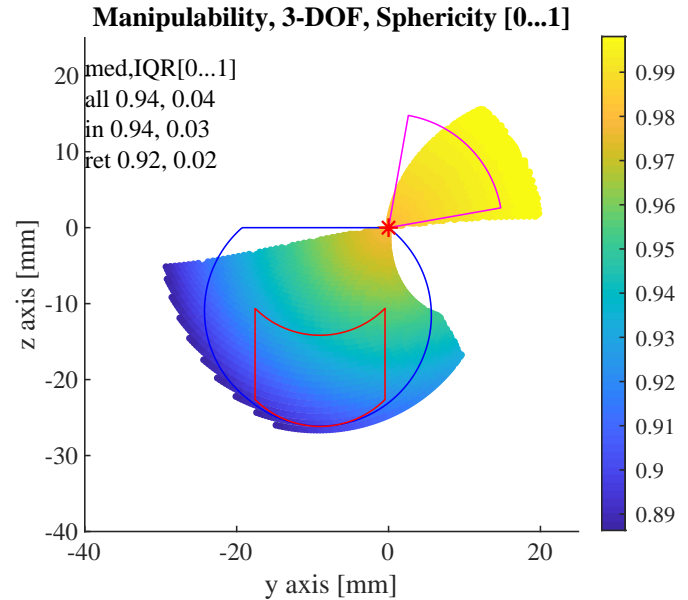

(b)

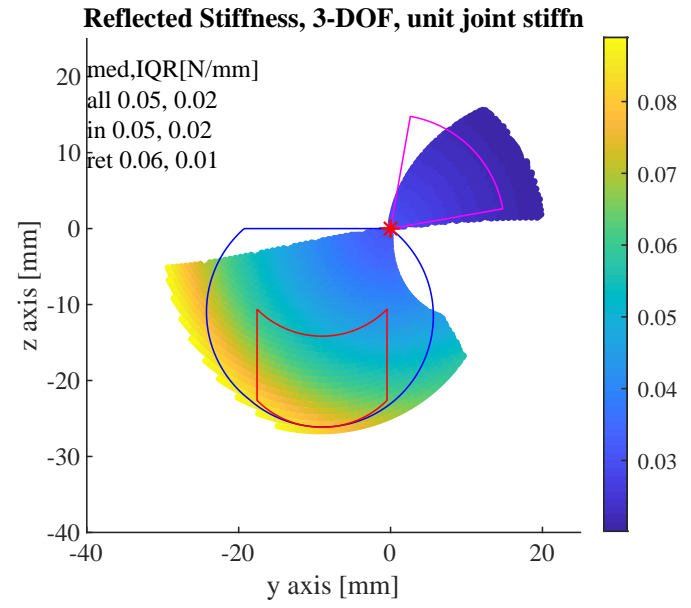

(d)

Figure 10: Each reviewed metric visualized for the found optimal kinematic design. More specifically (a) workspace coverage, (b) sphericity of manipulability, (c) planar potential energy, (d) reflected stiffness. Related zone-specific scalars are detailed in each top left corner. It should be noted that these metrics rely on design assumptions which may deviate from a final prototype design. As such, their primary purpose is to provide a means for relative comparison between kinematic designs within the optimal design algorithm. 
- from the following comparison. Furthermore, the comparison is based on median and interquartile range values for metrics across two difference workspace volumes. As such, equal performance in terms of metrics could still be considered an improvement given its presence across a larger workspace volume. Firstly, in terms of isotropic manipulability, both mechanisms present comparable median values, with overall less variation across the workspace for the newly proposed mechanism. This is believed to be beneficial. Secondly, the planar potential energy term shows overall less variation. Under the assumption that linkage crosssections are equal between mechanisms, the newly proposed architecture would require less potential energy to be counteracted from a gravity compensation solution. As such, this would allow for smaller passive compensation masses or lower-powered actuators to be used. Thirdly, a reduction in median reflected joint stiffness presents itself. This would seem to be mainly attributed to the increased linkage lengths of the rear parallelograms, as such highlighting a design trade-off between increased workspace and reduced reflected stiffness. The impact of this is to be validated in a later design phase than the current kinematic synthesis, however, care should be taken during the dimensioning of actuators and transmissions to meet a given mechanism stiffness requirement.

\section{Discussion}

\subsection{Mechanism}

This work builds further on prior work by Gijbels et al. 28, in which a generalized set of design rules is introduced to derive 2-DoF planar RCM mechanisms based on virtual parallelograms. Through the further adaptation of one of the conceptual embodiments proposed in [28, an improved 2-DoF planar RCM mechanism is presented and analysed. When compared to the state-of-the-art on 2-DoF planar RCM mechanisms, it addresses the following three concerns. First, the need for joints coinciding with the instrument axis is omitted, thus reducing hinder-some volume and mass at the end-effector to a minimum. As a result, this broadens options for instrument holder design and simplifies compatibility with existing surgical instruments. Second, mechanism complexity is maintained both in terms of the number of joints and linkages. The presented 2-DoF planar RCM mechanism combines 9 rigid bodies and a base, relying on a total of 14 rotative and 1 translational DoF implemented at 10 physical joint locations. As such, making it among the least complex 2-DoF planar RCM mechanism be synthesized in literature when compared to the identified state-of-the-art. Third, the need for a potentially harmful downward protruding link is omitted, thus improving inherent mechanism safety. While inherently safer, it should be noted that such a risk is also easily mitigated via the usage of a covering or protective frame, at the expense of limiting the placement of the mechanism in close proximity to the patient. Additionally, this leads to longer front parallelogram linkages, which in turn adversely affects mechanism stiffness or inertia. Given the absence of a downward protruding link, these design trade-off's are avoided with the proposed mechanism design.

\subsection{Algorithm}

Various algorithmic approaches have been reported on in literature, during which a specific performance aspect of a mechanism is optimised such as workspace coverage [36], isotropic manipulability 33, or a combination of both [37. While these studies identified a kinematic design by optimizing one or several mechanism performance aspects, these did so by reviewing these metrics across the entire workspace. For specific use-cases, it may be opportune to identify zones of interest within a desired workspace.

The algorithmic approach presented in this work combined the reviewal of multiple performance aspects with their reviewal within pre-defined zones of interest of an overall desired workspace. As such, optimisation of parameters can be restricted to these zones of interest, or assigned different weights dependant on the zone of interest it is reviewed in. For the given use case of vitreoretinal surgery, this was done by defining three areas of interest being: extraocular, intraocular, and retinal. By specifying minimal workspace requirements for each zone, these were used for a first identification of acceptable kinematic designs. Subsequently, this group of acceptable designs was used to identify an optimal kinematic design by reviewing several zonespecific performance parameters within a cost function. 


\subsection{Limitations}

The presented analysis and resulting outcome should be interpreted within its own limitations. First, the amount of reachable workspace outside the areas of interest is not quantified in the algorithm. Including this information could provide an alternative approach to optimize mechanism size for the desired reachable workspace, for instance by penalizing this excess workspace.

Furthermore, the current workspace analysis assumes that all intraocular zones are to be reached while the eye remains in an upward gazing pose. As such, it does not take into account the ability to rotate the eye, which is a common technique for visualisation during conventional retinal surgery. Using a robotic system, repositioning the RCM during surgery would allows for more eye anatomy to be reached without increasing the mechanism workspace [4]. Including these considerations may ground a less conservative set of workspace requirements, which may lead to a more compact mechanism design.

Also, the implemented review of the potential energy variation of the planar mechanism is limited, as it is bound to reasonable assumptions on linkage cross-section and COM location. While this approach provides insight into the amount of energy to be compensated, it only offers a relative form of comparison between kinematic designs. A revised analysis based on the final linkage geometries is required to design the gravity compensation.

It should be noted that overall mechanism stiffness, even though being an important design factor for cooperative systems, is only partially modelled in the current algorithm. Currently, stiffness is optimized only based on the reflected joint stiffness, as such leaving out structural compliance of the mechanism itself. In order to include this for comparison between kinematic designs, a parametric stiffness model similar to [42, 43] could be adopted in combination with reasonable assumptions on linkage stiffness. However, this is deemed out of scope at the time of writing. Furthermore, given a relatively large amount of design freedom in terms of material choice and linkage cross-sections, review of mechanism compliance is deemed more opportune in a later design phase - for instance via an FEA of the final conceptual design - for this use case.

The derived expression for manipulability is similar to the mechanism analysis method based on inertia ellipsoids as suggested by Yoshikawa et al. 44. The difference lies in the origin of the generated forces, which in the case of inertia ellipsoids are based on acceleration inputs and system inertia, opposed to the approach presented in this paper which relies on velocity inputs and system impedance. It is assumed that the forces due to linkage inertia are of lesser importance in the intended use-case, as the foreseeable surgical procedures are conducted at low accelerations. Other, more dynamic, use-cases could benefit from the addition of inertia based manipulability analysis to the presented numeric method.

\subsection{Clustering}

The current numerical approach provides a means to derive an optimal set of design parameters based on selected performance metrics. Kinematic constraints are taken into account at both joint and mechanism level. This approach is sufficient when a high certainty is present on the validity of the applied design constraints, as well as the achievability of exactly reproducing the determined optimal parameters. However, for design problems with remaining uncertainty on either the imposed constraints or the achievability of parameters, a review of parameter robustness could be beneficial. In these cases, a dense cluster of kinematic designs with acceptable parameters might be more desirable than a single optimal point at an early design phase, as configuration performance would be less affected by slight parameter changes. This could be done as follows: For a design case with n-parameters, an n-dimensional parameter space is defined. For each point within the parameter space, the parameter-configured system is evaluated on chosen performance metrics, resulting in a weighted score for each point in the parameter space. At this point, a clustering algorithm can be used to determine n-dimensional clusters of high-scoring system parameters, referred to as design zones. Now, a design zone can be selected based on the desired trade-off between system performance and parameter sensitivity.

\section{Conclusions}

This work reports on the synthesis of a parallel RCM mechanism, and its optimal design for the usecase of vitreoretinal surgery. A 2-DoF planar RCM mechanism is proposed, removing the need for joints 
coinciding with the instrument axis while maintaining remote actuation of the translational RCM DoF. When compared to the identified state-of-the-art, the proposed solution advantageously removes volume and mass at the end-effector and broadens options for instrument holder design. These improvements pose added value in surgical robot design and seek to further facilitate the real-world application of these mechanisms during robot-assisted surgery.

The 2-DoF mechanism is described and synthesised as part of a conceptual 4-DoF RCM mechanism, during which the inverse, forward, differential kinematics, and singularities are detailed. Subsequently, the 4-DoF RCM mechanism is designed for the use-case of vitreoretinal surgery, for which a numeric optimal

450 design algorithm is proposed and implemented. The workspace is determined within predefined kinematic constraints, after which key parameters such as workspace coverage, potential energy, manipulability, reflected stiffness are determined for specific interest zones within the available workspace. Once known, these parameters in combined in a score function, and used for finding an optimal kinematic design.

The resulting optimal configuration is briefly detailed and compared with the closely-related prior art which was designed for the same use-case. Initial results highlight improvements in relevant workspace, less required effort for passive gravity compensation, and more isotropic manipulability. Overall reflected stiffness is reduced and should be taken into account in future design phases.

Future work includes the further development of the presented kinematics into a first design and prototype, within a more detailed set of design requirements for the presented use-case. Furthermore, the presented optimal design algorithm could be expanded via additional modelling of properties such as passive stiffness and inertia. Finally, the implementation of alternative optimisation approaches such as identifying clusters of acceptable configurations could be explored.

\section{Acknowledgements}

Jonas Smits is a holder of an SB Fellowship of the Research Foundation - Flanders (1S41517N).

[1] A. M. Lacy, S. Delgado, A. Castells, H. A. Prins, V. Arroyo, A. Ibarzabal, J. M. Pique, The Long-term Results of a Randomized Clinical Trial of Laparoscopy-assisted Versus Open Surgery for Colon Cancer, Annals of Surgery 248 (1) (2008) 1-7. doi:10.1097/SLA.0b013e31816a9d65

[2] T. de Rooij, J. van Hilst, H. van Santvoort, D. Boerma, P. van den Boezem, F. Daams, R. van Dam, C. Dejong, E. van Duyn, M. Dijkgraaf, C. van Eijck, S. Festen, M. Gerhards, B. Groot Koerkamp, I. de Hingh, G. Kazemier, J. Klaase, R. de Kleine, C. van Laarhoven, M. Luyer, G. Patijn, P. Steenvoorde, M. Suker, M. Abu Hilal, O. Busch, M. Besselink, Minimally Invasive Versus Open Distal Pancreatectomy (LEOPARD), Annals of Surgery 269 (1) (2019) 2-9. doi:10.1097/SLA.0000000000002979

[3] C.-H. Kuo, J. S. Dai, P. Dasgupta, Kinematic design considerations for minimally invasive surgical robots: an overview, The International Journal of Medical Robotics and Computer Assisted Surgery 8 (2) (2012) 127-145. doi:10.1002/rcs.453

[ [4] R. H. Taylor, J. Funda, D. D. Grossman, J. P. Karidis, D. A. LaRose, US Patent 5397323 Remote center-of-motion robot for surgery (oct 1995).

[5] R. Beira, L. Santos-Carreras, G. Rognini, H. Bleuler, R. Clavel, Dionis: A novel remote-center-of-motion parallel manipulator for Minimally Invasive Surgery, Applied Bionics and Biomechanics 8 (2011) 191-208. doi:10.3233/ABB-2011-0020

480 [6] M. Hadavand, M. D. Naish, R. V. Patel, A parallel Remote Center of Motion mechanism for needle-based medical interventions, in: 5th IEEE RAS/EMBS International Conference on Biomedical Robotics and Biomechatronics, IEEE, 2014, pp. 1-6. doi:10.1109/BIOROB.2014.6913742

[7] D. Stoianovici, C. Jun, S. Lim, P. Li, D. Petrisor, S. Fricke, K. Sharma, K. Cleary, Multi-Imager Compatible, MR Safe, Remote Center of Motion Needle-Guide Robot IEEE Transactions on Biomedical Engineering 65 (1) (2018) 165-177. doi:10.1109/TBME. 2017.2697766

[8] B. Hannaford, J. Rosen, D. W. Friedman, H. King, P. Roan, L. Cheng, D. Glozman, J. Ma, S. N. Kosari, L. White, Raven-II: An open platform for surgical robotics research, IEEE Transactions on Biomedical Engineering 60 (4) (2013) 954-959. doi:10.1109/TBME.2012.2228858

[9] E. Vander Poorten, C. N. Riviere, J. J. Abbott, C. Bergeles, M. A. Nasseri, J. U. Kang, R. Sznitman, K. Faridpooya, I. Iordachita, Robotic Retinal Surgery, in: Handbook of Robotic and Image-Guided Surgery, Elsevier, 2020, pp. 627-672. doi:10.1016/B978-0-12-814245-5.00036-0

[10] J. Xiao, Q. Wu, D. Sun, C. He, Y. Chen, Classifications and Functions of Vitreoretinal Surgery Assisted Robots-A Review of the State of the Art, in: 2019 International Conference on Intelligent Transportation, Big Data \& Smart City (ICITBS), IEEE, 2019, pp. 474-484. doi:10.1109/ICITBS.2019.00122 
[11] E. Rahimy, J. Wilson, T.-C. Tsao, S. Schwartz, J.-P. Hubschman, Robot-assisted intraocular surgery: development of the IRISS and feasibility studies in an animal model., Eye (London, England) 27 (8) (2013). doi:10.1038/eye.2013.105.

[12] A. Guerrouad, P. Vidal, SMOS: stereotaxical microtelemanipulator for ocular surgery, in: Proceedings of the Annual International Engineering in Medicine and Biology Society, 1989, pp. 879-880. doi:10.1109/IEMBS.1989.96028

[13] D.-Y. Yu, S. Cringle, I. Constable, Robotic ocular ultramicrosurgery, Australian and New Zealand Journal of Ophthalmology 26 (1998) S6-S8.

[14] T. Meenink, Vitreo-retinal eye surgery robot : sustainable precision, Ph.D. thesis (2011). doi:10.6100/IR717725

[15] A. Mablekos-Alexiou, S. Ourselin, L. Da Cruz, C. Bergeles, Requirements Based Design and End-to-End Dynamic Modeling of a Robotic Tool for Vitreoretinal Surgery, in: Proceedings - IEEE International Conference on Robotics and Automation, Institute of Electrical and Electronics Engineers Inc., 2018, pp. 135-141. doi:10.1109/ICRA.2018.8460921

505 [16] A. Molaei, E. Abedloo, H. D. Taghirad, Z. Marvi, Kinematic and workspace analysis of DIAMOND: An innovative eye surgery robot, in: ICEE 2015 - Proceedings of the 23rd Iranian Conference on Electrical Engineering, Vol. 10, IEEE, 2015, pp. 882-887. doi:10.1109/IranianCEE. 2015.7146336

[17] X. He, D. Roppenecker, D. Gierlach, M. Balicki, K. Olds, P. Gehlbach, J. Handa, R. Taylor, I. Iordachita, Toward Clinically Applicable Steady-Hand Eye Robot for Vitreoretinal Surgery in: Proceedings of the ASME 2012 International Mechanical Engineering Congress \& Exposition (IMECE2012), no. 88384, 2012, pp. 145-153.

[18] T. Nakano, N. Sugita, T. Ueta, Y. Tamaki, M. Mitsuishi, A parallel robot to assist vitreoretinal surgery, International Journal of Computer Assisted Radiology and Surgery 4 (6) (2009) 517-526. doi:10.1007/s11548-009-0374-2

[19] P. S. Schenker, H. Das, T. R. Ohm, A New Robot for High Dexterity Microsurgery, in: Computer Vision Virtual Reality and Robotics in Medicine, Vol. 905, 1995, pp. 115-122. doi:10.1007/BFb0034939

20] M. A. Nasseri, M. Eder, S. Nair, E. C. Dean, M. Maier, D. Zapp, C. P. Lohmann, A. Knoll, The Introduction of a New Robot for Assistance in Ophthalmic Surgery, in: Annual International Conference of the IEEE Engineering in Medicine and Biology Society, Vol. 35, IEEE, 2013, pp. 5682-5. doi:10.1109/EMBC.2013.6610840.

[21] M. Nambi, P. S. Bernstein, J. J. Abbott, A Compact Retinal-Surgery Telemanipulator that Uses Disposable Instruments Springer, Cham, 2015, pp. 258-265. doi:10.1007/978-3-319-24553-9_32

$22]$ B. Mitchell, J. Koo, I. Iordachita, P. Kazanzides, A. Kapoor, J. Handa, G. Hager, R. Taylor, Development and application of a new steady-hand manipulator for retinal surgery, in: Proceedings - IEEE International Conference on Robotics and Automation, no. April, 2007, pp. 623-629. doi:10.1109/ROBOT.2007.363056

[23] C.-Y. He, L. Huang, Y. Yang, Q.-F. Liang, Y.-K. Li, Research and Realization of a Master-Slave Robotic System for Retinal Vascular Bypass Surgery Chinese Journal of Mechanical Engineering 31 (1) (2018) 78. doi:10.1186/s10033-018-0278-6

[24] A. Gijbels, J. Smits, L. Schoevaerdts, K. Willekens, E. B. Vander Poorten, P. Stalmans, D. Reynaerts, In-Human RobotAssisted Retinal Vein Cannulation, A World First, Annals of Biomedical Engineering 46 (10) (2018) 1676-1685. doi: 10.1007/s10439-018-2053-3

[25] A. Gijbels, N. Wouters, P. Stalmans, H. Van Brussel, D. Reynaerts, E. V. Poorten, Design and realisation of a novel robotic manipulator for retinal surgery in: 2013 IEEE/RSJ International Conference on Intelligent Robots and Systems, IEEE, 2013, pp. 3598-3603. doi:10.1109/IROS.2013.6696869.

[26] J. Smits, A. Gijbels, K. Willekens, B. V. Stanzel, D. Reynaerts, Robot-Assisted Subretinal Surgery: initial in-vivo animal validation, in: Proceedings of the 11th Hamlyn Symposium on Medical Robotics, 2018, pp. 91-92.

[27] M. Ourak, J. Smits, L. Esteveny, G. Borghesan, A. Gijbels, L. Schoevaerdts, Y. Douven, J. Scholtes, E. Lankenau, T. Eixmann, H. Schulz-Hildebrandt, G. Hüttmann, M. Kozlovszky, G. Kronreif, K. Willekens, P. Stalmans, K. Faridpooya, M. Cereda, A. Giani, G. Staurenghi, D. Reynaerts, E. B. Vander Poorten, Combined OCT distance and FBG force sensing cannulation needle for retinal vein cannulation: in vivo animal validation, International Journal of Computer Assisted Radiology and Surgery (2018) 1-9doi:10.1007/s11548-018-1829-0

[28] A. Gijbels, D. Reynaerts, E. B. Vander Poorten, Design of 4-DOF Parallelogram-Based RCM Mechanisms with a Translational DOF Implemented Distal from the End-Effector in: Advances on Theory and Practice of Robots and Manipulators: Proceedings of Romansy 2014 XX CISM-IFToMM Symposium on Theory and Practice of Robots and Manipulators, 2014, pp. 103-111. doi:10.1007/978-3-319-07058-2_12.

[29] E. B. Vander Poorten, A. Gijbels, N. Wouters, WO2014108545 (A1) - An apparatus for generating motion around a remote centre of motion (jan 2014).

[30] H. Long, Y. Yang, X. Jingjing, S. Peng, Type Synthesis of 1R1T Remote Center of Motion Mechanisms Based on Pantograph Mechanisms Journal of Mechanical Design 138 (1) (jan 2016). doi:10.1115/1.4031804

[31] J. Li, G. Zhang, Y. Xing, H. Liu, S. Wang, A Class of 2-Degree-of-Freedom Planar Remote Center-of-Motion Mechanisms Based on Virtual Parallelograms Journal of Mechanisms and Robotics 6 (3) (2014) 031014. doi:10.1115/1.4027239

[32] S. Nisar, T. Endo, F. Matsuno, Design and Kinematic Optimization of a Two Degrees-of-Freedom Planar Remote Center of Motion Mechanism for Minimally Invasive Surgery Manipulators Journal of Mechanisms and Robotics 9 (3) (2017) 031013. doi:10.1115/1.4035991.

33] S. Nisar, T. Endo, F. Matsuno, Design and optimization of a 2-degree-of-freedom planar remote center of motion mechanism for surgical manipulators with smaller footprint Mechanism and Machine Theory 129 (2018) 148-161. doi:10.1016/j. mechmachtheory.2018.07.020

[34] G. Chen, J. Wang, H. Wang, A New Type of Planar Two Degree-of-Freedom Remote Center-of-Motion Mechanism Inspired by the Peaucellier-Lipkin Straight-Line Linkage Journal of Mechanical Design 141 (1) (jan 2019). doi:10.1115/1.4041221

[35] J.-P. Merlet, C. Gosselin, Parallel Mechanisms and Robots in: Springer Handbook of Robotics, Springer Berlin Heidelberg, Berlin, Heidelberg, 2008, pp. 269-285. doi:10.1007/978-3-540-30301-5_13.

[36] Y. Lou, G. Liu, J. Xu, Z. Li, A general approach for optimal kinematic design of parallel manipulators in: IEEE International Conference on Robotics and Automation, 2004. Proceedings. ICRA '04. 2004, IEEE, 2004, pp. 3659-3664 
[37] M. Lum, J. Rosen, M. Sinanan, Hannaford B, Kinematic optimization of a spherical mechanism for a minimally invasive surgical robot, in: IEEE International Conference on Robotics and Automation, 2004. Proceedings. ICRA '04. 2004, no. April, IEEE, 2004, pp. 829-834 Vol.1. doi:10.1109/ROBOT.2004.1307252

[38] G. Hamlin, A. Sanderson, A novel concentric multilink spherical joint with parallel robotics applications in: Proceedings of the 1994 IEEE International Conference on Robotics and Automation, no. pt 2, IEEE Comput. Soc. Press, 1994, pp. 1267-1272. doi:10.1109/ROBOT.1994.351313

[39] F. Caccavale, M. Uchiyama, Cooperative Manipulators, in: Springer Handbook of Robotics, Springer Berlin Heidelberg, Berlin, Heidelberg, 2008, pp. 701-718. doi:10.1007/978-3-540-30301-5_30

[40] J. P. Merlet, Jacobian, Manipulability, Condition Number, and Accuracy of Parallel Robots, Journal of Mechanical Design 128 (1) (2006) 199-206. doi:10.1115/1.2121740

J. Smits, D. Reynaerts, E. Vander Poorten, Setup and Method for Remote Center of Motion Positioning Guidance During Robot-Assisted Surgery, in: Proceedings of the 2019 IEEE/RSJ International Conference on Intelligent Robots and Systems (IROS), 2019, pp. 1315-1322.

[42] O. Company, F. Pierrot, Modelling and design issues of a 3-axis parallel machine-tool Mechanism and Machine Theory 37 (11) (2002) 1325-1345. doi:10.1016/S0094-114X(02) 00040-X

[43] F. Majou, C. Gosselin, P. Wenger, D. Chablat, Parametric stiffness analysis of the Orthoglide, Mechanism and Machine Theory 42 (3) (2007) 296-311. arXiv:0708.3723, doi:10.1016/j.mechmachtheory.2006.03.018

[44] T. Yoshikawa, Manipulability of Robotic Mechanisms, The International Journal of Robotics Research 4 (2) (1985) 3-9. doi: $10.1177 / 027836498500400201$

\section{Appendix A. Detailed expressions Jacobian matrix}

$$
\begin{aligned}
J_{1,2} & =-\frac{l_{15} \sigma_{2}}{\sigma_{4}} \\
J_{1,3} & =-\frac{l_{16} \sigma_{1}}{\sigma_{4}} \\
J_{2,2} & =\frac{l_{15}\left(l_{15}-l_{8} \cos \left(q_{2}\right)+l_{13} \cos \left(q_{2}\right)-l_{14} \sin \left(q_{2}\right)+l_{16} \cos \left(q_{2}-q_{3}\right)\right)}{\sigma_{11}+\sigma_{12}}-\ldots \\
& \frac{\frac{l_{15} \sigma_{2} \sigma_{8}}{\sigma_{5}}+\frac{l_{15} \sigma_{2} \sigma_{8}}{\sigma_{6}}-\frac{l_{15}\left(2 \sigma_{9}-\sigma_{10}\right) \sigma_{2}}{\sigma_{7}}}{\sigma_{3}} \\
J_{2,3} & =\frac{l_{16}\left(l_{16}-l_{8} \cos \left(q_{3}\right)+l_{13} \cos \left(q_{3}\right)-l_{14} \sin \left(q_{3}\right)+l_{15} \cos \left(q_{2}-q_{3}\right)\right)}{\sigma_{11}+\sigma_{12}}-\ldots
\end{aligned}
$$


where

$$
\begin{aligned}
& l_{45}=l_{4}+l_{5} \\
& \sigma_{1}=l_{14} \cos \left(q_{3}\right)-l_{8} \sin \left(q_{3}\right)+l_{13} \sin \left(q_{3}\right)-l_{15} \sin \left(q_{2}-q_{3}\right) \\
& \sigma_{2}=l_{14} \cos \left(q_{2}\right)-l_{8} \sin \left(q_{2}\right)+l_{13} \sin \left(q_{2}\right)+l_{16} \sin \left(q_{2}-q_{3}\right) \\
& \sigma_{3}=\sqrt{1-\frac{\sigma_{8}^{2}}{\left(4 \sigma_{11}+4 \sigma_{12}\right)\left(\sigma_{9}-\sigma_{10}\right)^{2}}} \\
& \sigma_{4}=\sqrt{\sigma_{11}+l_{45}{ }^{2} \cos (\alpha)^{2}-l_{6}{ }^{2}-l_{45}{ }^{2}+\sigma_{12}-\sigma_{13}} \\
& \sigma_{5}=2\left(\sigma_{11}+\sigma_{12}\right)^{3 / 2}\left(\sigma_{9}-\sigma_{10}\right) \\
& \sigma_{6}=2 \sqrt{\sigma_{11}+\sigma_{12}}\left(\sigma_{9}-\sigma_{10}\right)^{2} \sigma_{9} \\
& \sigma_{7}=\sqrt{\sigma_{11}+\sigma_{12}}\left(\sigma_{9}-\sigma_{10}\right) \sigma_{9} \\
& \sigma_{8}=\sigma_{11}+\left(\sigma_{9}-\sigma_{10}\right)^{2}-l_{6}{ }^{2}-l_{45}{ }^{2}+\sigma_{12}-\sigma_{13} \\
& \sigma_{9}=\sqrt{\sigma_{11}-l_{45}^{2} \sin (\alpha)^{2}-l_{6}^{2}+\sigma_{12}-\sigma_{13}} \\
& \sigma_{10}=\sqrt{\frac{l_{45}^{2}}{\sigma_{14}}} \sqrt{1-\sin (\alpha)^{2}} \sqrt{\sigma_{14}} \\
& \sigma_{11}=\left(l_{13}-l_{8}+l_{15} \cos \left(q_{2}\right)+l_{16} \cos \left(q_{3}\right)\right)^{2} \\
& \sigma_{12}=\left(l_{15} \sin \left(q_{2}\right)-l_{14}+l_{16} \sin \left(q_{3}\right)\right)^{2} \\
& \sigma_{13}=2 l_{6} l_{45} \sin (\alpha) \\
& \sigma_{14}=l_{6}^{2}+2 \sin (\alpha) l_{6} l_{45}+l_{45}^{2}
\end{aligned}
$$

